Article

\title{
The Role of Substitution in the Apex Position of the Bent-Core on Mesomorphic Properties of New Series of Liquid Crystalline Materials
}

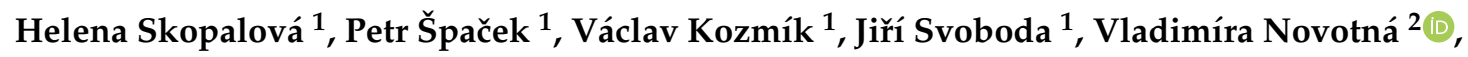 \\ Damian Pociecha $^{3}$ (D) and Michal Kohout ${ }^{1, *(D)}$ \\ 1 Department of Organic Chemistry, University of Chemistry and Technology, CZ-166 28 Prague 6, \\ Czech Republic; helena.skopalova@vscht.cz (H.S.); petr.spacek@uochb.cas.cz (P.Š.); \\ vaclav.kozmik@vscht.cz (V.K.); jiri.svoboda@vscht.cz (J.S.) \\ 2 Institute of Physics of the Czech Academy of Sciences, Na Slovance 2, CZ-182 21 Prague 9, Czech Republic; \\ novotna@fzu.cz \\ 3 Laboratory of Dielectrics and Magnetics, Chemistry Department, Warsaw University, \\ Al. Zwirki i Wigury 101, 02-089 Warsaw, Poland; pociu@chem.uw.edu.pl \\ * Correspondence: michal.kohout@vscht.cz; Tel.: +420-220444118
}

Received: 31 July 2020; Accepted: 18 August 2020; Published: 21 August 2020

check for updates

\begin{abstract}
We present the synthesis and mesomorphic properties of the new series of bent-core liquid crystals based on 3-hydroxybenzoic acid bearing a lateral substituent in the apex position. Four different substituents of various sizes and electronic properties have been used. We have found that only compounds substituted with fluorine are mesogenic and exhibit one mesophase, whose type differs when prolonging the terminal alkyl chain. For homologues with shorter alkyl chains (octyl, decyl), a columnar $B_{1}$-type of a mesophase was observed, while materials with longer terminal chains (dodecyl, tetradecyl) exhibited a switchable lamellar $\mathrm{SmC}_{\mathrm{A}} \mathrm{P}_{\mathrm{A}}$ phase. Calorimetric measurements, texture observations under a polarizing microscope were performed and electro-optical properties studied. Additionally, dielectric measurements were realized to characterize the molecular dynamics in the $\mathrm{SmC}_{\mathrm{A}} \mathrm{P}_{\mathrm{A}}$ phase. All mesogenic compounds were further studied by $\mathrm{X}$-ray measurements to confirm phase identification and obtain more information about their structural parameters.
\end{abstract}

Keywords: liquid crystals; bent-core; columnar phase; switchable lamellar phase; dielectric spectroscopy

\section{Introduction}

Bent-core materials represent a unique class of liquid crystals (LCs). The first compounds of this type were already synthesized at the beginning of the 20th century [1]. However, they were not studied more widely due to the low thermal stability of formed mesophases. They were rediscovered in the 1990s, when the polar character and macroscopic chirality of mesophases formed exclusively by bent-core materials were described [2,3]. Since then, many unique features of bent-core LCs have been discovered. Bent-core compounds can form a broad variety of mesophases ranging from low-organized nematic phases over various lamellar mesophases to structurally complex ones [4]. The nematic phase of bent-core LCs has attracted much attention due to its possible practical applications [5]. In particular, the biaxial nematic phase formed by bent molecules has been the aim of extensive research, since it could be useful for the construction of a new generation of liquid crystal displays [6,7]. The polar and chiral orders of nematic phases formed by bent-core LCs are nowadays of great interest [8-10] as well as their modulation with light stimulus, which can be used for the construction of diffraction devices [11]. 
Polar order with the ability of symmetry breaking is a typical feature of lamellar and columnar phases formed by bent-core LCs [12,13]. The most typical example of a lamellar phase is a tilted polar smectic $C$ phase $(\mathrm{SmCP})$, where the tilt correlation can be synclinic $\left(\mathrm{SmC}_{\mathrm{S}}\right)$ or anticlinic $\left(\mathrm{SmC}_{\mathrm{A}}\right)$ and the correlation between adjacent layers can be either ferroelectric $\left(\mathrm{P}_{\mathrm{F}}\right)$ or antiferroelectric $\left(\mathrm{P}_{\mathrm{A}}\right)$ leading to four possible molecular arrangements: $\mathrm{SmC}_{\mathrm{S}} \mathrm{P}_{\mathrm{F}}, \mathrm{SmC}_{\mathrm{S}} \mathrm{P}_{\mathrm{A}}, \mathrm{SmC}_{\mathrm{A}} \mathrm{P}_{\mathrm{F}}, \mathrm{SmC}_{\mathrm{A}} \mathrm{P}_{\mathrm{A}}$. From these four states, $\mathrm{SmC}_{\mathrm{S}} \mathrm{P}_{\mathrm{F}}$ and $\mathrm{SmC}_{\mathrm{A}} \mathrm{P}_{\mathrm{A}}$ are chiral and for each of them an enantiomeric state exists. The ability of $\mathrm{SmCP}$ phases to switch under an applied electric field then offers an interesting option for electro-optical applications $[2,12,14,15]$. Two basic structures were proposed for columnar mesophases and the nomenclature $B_{1}$ and $B_{1 \text { Rev }}$ was utilized $[15,16]$. The columnar mesophases can be described as blocks of molecular layers. For the $B_{1}$ phase the modulation exists in the plane parallel to the polarization vector, $\mathrm{P}$, and no electro-optic response or switching current has been detected. In the case of the modulation plane perpendicular to the $\mathrm{P}$ vector, designation $\mathrm{B}_{1 \mathrm{Rev}}$ was proposed. Both columnar structures can exist with tilted or nontilted molecules with respect to the layer normal.

The molecule of a bent-core LC is a dynamic system in which even a tiny structural modification can lead to different physical properties. The central aromatic unit significantly contributes to the stabilization of mesomorphic behavior by means of $\pi-\pi$-interactions increasing the size of the central core leading to the preferential formation of columnar phases [16]. The side arms connected to the central core (typically with the included angle of $120^{\circ}$ ) feature polar-linking groups. The character and orientation of these linking units contribute to the overall polarity and dipole moment of the molecule. For resorcinol-based materials, it was documented that mesomorphic properties significantly varied, depending on the orientation of the ester groups in the side arms [17]. A similar trend was observed also for naphthalene-based materials [18-20]. For resorcinol as well as naphthalene-based materials, terminal alkyl chains were used to stabilize a lamellar phase over a columnar phase. Generally, homologues with short alkyl chains (up to $\mathrm{C}_{10} \mathrm{H}_{21}$ ) formed columnar phases, while materials bearing longer terminal alkyl chains exhibited smectic phases (SmCP). This is due to a higher increment of London dispersion forces of alkyl chains, which in the case of longer chains prevail over the effect of the $\pi-\pi$-interactions of the aromatic units. A similar effect can be induced by the introduction of siloxane or perfluoroalkyl chains, where the nanosegregation of fluorinated (siloxane) and alkyl chains leads to preferential formation of lamellar-ordered phases [21,22].

Lateral substituents connected to the central core, or the aromatic units in the side chains, play an important role in tuning the mesomorphic properties of bent-core LCs. A number of studies on the influence of lateral substituents on mesomorphic behavior in terms of their steric and electronic effect in various positions of aromatic units of bent-core materials have been published thus far [23-31]. In our preceding studies, we have systematically investigated the effect of lateral substituents connected in different positions to a central unit of 3-hydroxybenzoic acid [32-35]. We observed the preferential formation of a nematic phase for materials with lateral substituent $\left(\mathrm{F}, \mathrm{Cl}, \mathrm{CH}_{3}\right)$ in position four of the central unit. This tendency was tuned by the orientation and number of the linking ester units. Materials with a uniform orientation of five ester units formed columnar $\mathrm{B}_{1 \mathrm{Rev}}$, polar SmA (SmAP) phase or $\mathrm{SmC}_{S} \mathrm{P}_{\mathrm{A}}$ [33]. A significant effect of lateral substituents was observed for six substituted materials, for which only columnar and lamellar phases were observed. With the increasing size of the substituent (i.e., for the chlorine and methyl groups) and the increasing number of ester-linking units with uniform orientation the materials became crystalline [34]. Surprisingly, positioning the lateral substituents between the side arms (in position two) did not substantially influence the mesomorphic behavior; materials exhibited a columnar $\mathrm{B}_{1}$ phase and lamellar $\mathrm{SmC}_{\mathrm{A}} \mathrm{P}_{\mathrm{A}}$ phase in the case of short $\left(\mathrm{C}_{8} \mathrm{H}_{17}\right.$ and $\left.\mathrm{C}_{10} \mathrm{H}_{21}\right)$ and long $\left(\mathrm{C}_{12} \mathrm{H}_{25}\right.$ and $\left.\mathrm{C}_{14} \mathrm{H}_{29}\right)$ terminal alkyl chains, respectively. Exceptional behavior was determined for methyl-substituted materials that showed a nematic phase as well as polymorphism and a sequence of nematic, $\mathrm{SmC}$ and $\mathrm{SmC}_{\mathrm{A}} \mathrm{P}_{\mathrm{A}}$ phases [35].

In this study, we have focused on materials bearing the lateral substitution in position five (the apex) of the central 3-hydroxybenzoic acid-based unit. In comparison to the previously studied 
materials, the lateral substituents $\left(\mathrm{F}, \mathrm{Cl}, \mathrm{CH}_{3}, \mathrm{NO}_{2}\right)$ used here have shown a strong steric effect on mesomorphic behavior.

\section{Materials and Methods}

\subsection{Synthesis of the Protected Central Cores}

Synthesis of the 5-fluoro (1) and 5-methyl-3-hydroxy protected benzoic acids (3) has been described elsewhere [36]. Preparation of the corresponding 5-chloro 2 and 5-nitro-3-hydroxy protected benzoic acid (4) is depicted in Scheme 1. While the hydroxylic group of the fluoro and methyl substituted central cores 1,3 was protected by benzyl group (Bn), for synthetic reasons protection of acids 2 and 4 was achieved by tert-butyldimethylsilyl group (TBS).

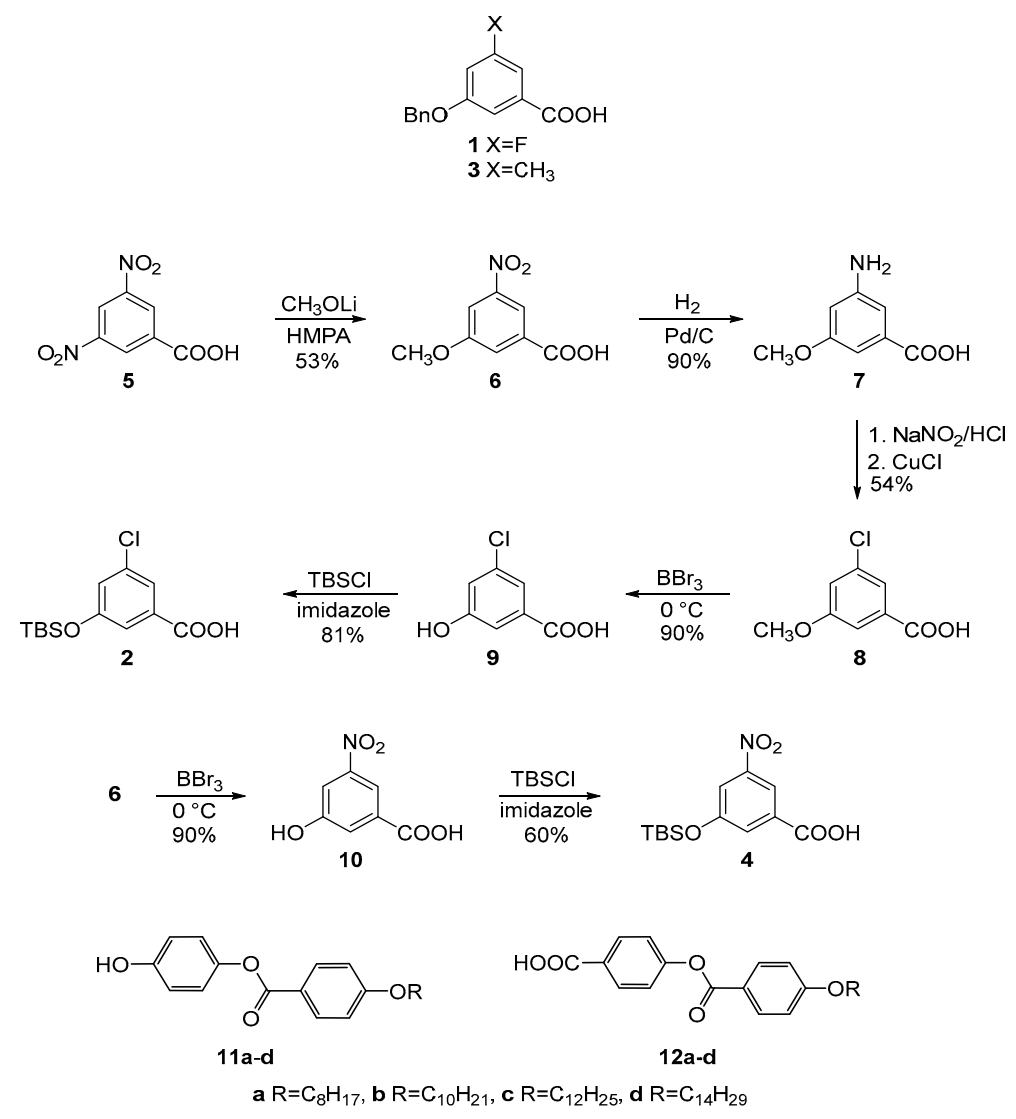

Scheme 1. Synthesis of the protected central cores 1-4.

The protected chloro acid 2 was obtained by a multistep transformation of 3,5-dinitrobenzoic acid (5). First, one of the nitro groups was substituted by the means of lithium methoxide in hexamethylphosphoric amide (HMPA) [37] to give rise to methoxy acid 6. The nitro group of $\mathbf{6}$ was then reduced by catalytic hydrogenation on $\mathrm{Pd} / \mathrm{C}$ to yield the corresponding amino acid 7 , see reference [38]. The amino group of 7 was subsequently diazotized and the diazonium group substituted in a standard Sandmeyer reaction to yield the chloro acid 8. Deprotection of the methoxy group was achieved with boron tribromide and the released hydroxylic group of $\mathbf{9}$ was finally reprotected by silylation with tert-butyldimethylsilyl chloride (TBSCl) to form the protected central core 2.

The intermediate methoxy nitro acid $\mathbf{6}$ served also for the synthesis of the nitro acid 4 . The methoxy group of $\mathbf{6}$ was first deprotected with boron tribromide to yield the hydroxy acid $\mathbf{1 0}$ and the free hydroxylic group was finally reprotected with TBSCl to provide the 5-nitro protected acid 4 . To introduce the lengthening arms, the known phenols 11a-d and acids 12a-d were used [20]. 


\subsection{Synthesis of the Target Materials}

The series of target materials Ia-d $(X=F)$, IIa-d $(X=C l)$, IIIa-d $\left(X=\mathrm{CH}_{3}\right)$, and IVa-d $\left(X=\mathrm{NO}_{2}\right)$ (Scheme 2) were prepared by the same methodology as previously [18-20]. First, the acids 1-4 were coupled with the substituted phenols 11a-d in the presence of $N, N^{\prime}$-dicyclohexylcarbodiimide (DCC) and $\mathrm{N}, \mathrm{N}$-dimethylaminopyridine (DMAP) as catalyst to yield the protected (PG = protecting group) intermediates $\mathbf{1 3} \mathbf{a}-\mathbf{d} \mathbf{- 1 6 a}-\mathbf{d}$. The protecting group was removed with respect to its character. While for the deprotection of the benzyl group ( $\mathrm{PG}=\mathrm{C}_{6} \mathrm{H}_{5} \mathrm{CH}_{2}$ ) transfer-hydrogenation using $\mathrm{Pd} / \mathrm{C}$ and ammonium formate was utilized, the silyl moiety $\left(\mathrm{PG}=\left(\mathrm{CH}_{3}\right)_{3} \mathrm{CSi}\left(\mathrm{CH}_{3}\right)_{2}\right)$ was removed by the means of tetrabutylammonium fluoride $\left(\mathrm{Bu}_{4} \mathrm{~N}^{+} \mathrm{F}^{-}\right)$in wet tetrahydrofuran [39]. In the last step, the hydroxy esters 17a-d-20a-d were acylated with acid chlorides of acids 12a-d in the presence of DMAP as a base yielding target compounds of series Ia-d $(\mathrm{X}=\mathrm{F}), \mathbf{I I a}-\mathbf{d}(\mathrm{X}=\mathrm{Cl})$, IIIa-d $\left(\mathrm{X}=\mathrm{CH}_{3}\right)$, and IVa-d $\left(\mathrm{X}=\mathrm{NO}_{2}\right)$, respectively.

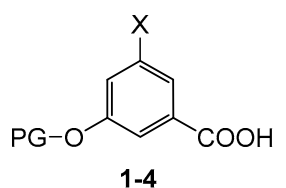

$$
\mid \begin{aligned}
& \text { 11a-d } \\
& \text { DCC } \\
& \text { DMAP }
\end{aligned}
$$

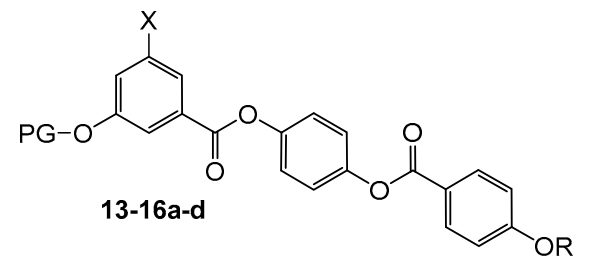

13a-d $X=F, 14 a-d X=C l, 15 a-d X=\mathrm{CH}_{3}, 16 a-d X=\mathrm{NO}_{2}$

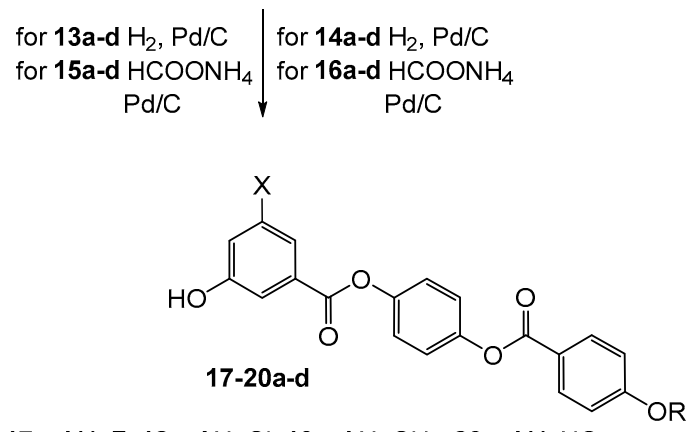

17a-d $X=F, 18 a-d X=C l, 19 a-d X=\mathrm{CH}_{3}, 20 a-d X=\mathrm{NO}_{2}$

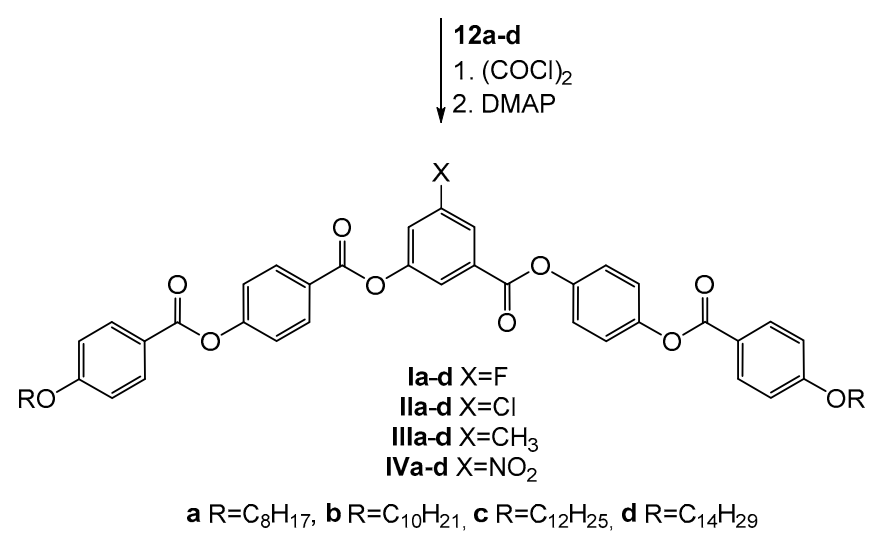

Scheme 2. General scheme of the synthesis of the target materials of series I-IV. 


\subsection{Synthesis of the Central Cores}

\section{3-Methoxy-5-nitrobenzoic acid (6)}

A mixture of dinitro acid 5 ( $49.0 \mathrm{~g} ; 231 \mathrm{mmol})$ and lithium methoxide ( $35.0 \mathrm{~g} ; 0.92 \mathrm{~mol})$ in freshly distilled hexamethylphosphoric amide (HMPA) $(600 \mathrm{~mL})$ was stirred in an inert argon atmosphere at room temperature for $18 \mathrm{~h}$ and at $80^{\circ} \mathrm{C}$ for $6 \mathrm{~h}$ until the starting compound disappeared (tlc). After cooling, the mixture was poured on ice $(600 \mathrm{~mL})$ and acidified with conc. hydrochloric acid $(200 \mathrm{~mL})$. The product was extracted with ether $(6 \times 600 \mathrm{~mL})$, the combined ethereal solution was washed with brine $(400 \mathrm{~mL})$, and dried with anhydrous magnesium sulphate. The solvent was removed to yield $44.6 \mathrm{~g}$ (98\%) of acid 6, m.p. $194-197^{\circ} \mathrm{C}, 187-189^{\circ} \mathrm{C}$ [37].

\section{3-Amino-5-methoxybenzoic acid (7)}

To a solution of acid 6 (29.3 g; $148 \mathrm{mmol})$ in ethyl acetate $(300 \mathrm{~mL}), 10 \% \mathrm{Pd} / \mathrm{C}(4.2 \mathrm{~g})$ was added and the mixture was hydrogenated in a Parr apparatus. The catalyst was filtered and the filtrate evaporated. Crystallisation from ethanol yielded $19.7 \mathrm{~g}(79 \%)$ of acid 7, m.p. $194-197^{\circ} \mathrm{C}$, m.p. $184^{\circ} \mathrm{C}$ [40].

\section{3-Chloro-5-methoxybenzoic acid (8)}

To a solution of 3-amino-5-methoxybenzoic acid (7) (4.98 g; $29.8 \mathrm{mmol})$ in $12 \%$ aq. $\mathrm{HCl}(60 \mathrm{~mL})$ cooled to $5{ }^{\circ} \mathrm{C}$, a solution of sodium nitrite $(2.59 \mathrm{~g} ; 37.5 \mathrm{mmol})$ in water $(20 \mathrm{ml})$ was added drop wise. The mixture was stirred at $0{ }^{\circ} \mathrm{C}$ for $30 \mathrm{~min}$, then a solution of copper (I) chloride ( $3.60 \mathrm{~g} ; 36.3 \mathrm{mmol}$ ) in $36 \%$ aq. $\mathrm{HCl}(20 \mathrm{~mL})$ was added to keep the temperature below $10^{\circ} \mathrm{C}$. The formed suspension was diluted with water and stirred at room temperature for $1 \mathrm{~h}$ and at $80^{\circ} \mathrm{C}$ for $30 \mathrm{~min}$. After cooling to room temperature, the product was filtered, washed with water $(2 \times 50 \mathrm{~mL})$, and dried at reduced pressure. After purification by a column chromatography (toluene/methanol/acetic acid 32/1/1), $3.02 \mathrm{~g}$ (54\%) of acid 8 was isolated, m.p. $182-184{ }^{\circ} \mathrm{C}, 170-171{ }^{\circ} \mathrm{C}$ [41].

\section{3-Chloro-5-hydroxybenzoic acid (9)}

To a solution of acid $8(3.20 \mathrm{~g} ; 17.1 \mathrm{mmol})$ in dichloromethane $(40 \mathrm{~mL})$ cooled to $-78{ }^{\circ} \mathrm{C}$, boron tribromide $(6.1 \mathrm{~mL} ; 65.2 \mathrm{mmol})$ was added drop wise in the argon atmosphere. The solution was stirred for $30 \mathrm{~min}$, then the cooling bath was removed, and the stirring continued at $0{ }^{\circ} \mathrm{C}$ for $6 \mathrm{~h}$. The mixture was poured on ice $(300 \mathrm{~mL})$ and after decomposition of the reagent extracted with ethyl acetate $(3 \times 150 \mathrm{~mL})$. The organic solution was then washed with brine $(150 \mathrm{~mL})$ and dried with anhydrous magnesium sulphate. After evaporation of the solvent, the crude product was purified by column chromatography (toluene/methanol/acetic acid 32/1/1) to yield $2.67 \mathrm{~g}(90 \%)$ of 9, m.p. $213-216^{\circ} \mathrm{C}$. ${ }^{1} \mathrm{H}$ NMR spectrum (acetone- $\left.d_{6}\right): 2.82(\mathrm{~s}, 1 \mathrm{H}, \mathrm{OH}), 7.11(\mathrm{~m}, 1 \mathrm{H}), 7.45(\mathrm{~m}, 1 \mathrm{H}), 7.48(\mathrm{~m}, 1 \mathrm{H}), 9.18(\mathrm{~s}, 1 \mathrm{H}$, $\mathrm{COOH}$ ). Elemental analysis: for $\mathrm{C}_{7} \mathrm{H}_{5} \mathrm{ClO}_{3}$ (172.57): calculated $\mathrm{C} 48.72, \mathrm{H} 2.92, \mathrm{Cl} 20.54$; found $\mathrm{C} 48.55$, $\mathrm{H} 2.82, \mathrm{Cl} 20.36 \%$.

\section{3-(tert-Butyldimethylsilyloxy)-5-chlorobenzoic acid (2)}

To a solution of acid 9 ( $2.67 \mathrm{~g} ; 15.5 \mathrm{mmol})$ and imidazole $(3.21 \mathrm{~g} ; 47.2 \mathrm{mmol})$ in dry DMF (45 mL), a solution of tert-butyldimethylchlorosilane $(7.08 \mathrm{~g} ; 46.9 \mathrm{mmol})$ in dry DMF $(45 \mathrm{~mL})$ was added in an argon atmosphere and the mixture was stirred and heated to $60^{\circ} \mathrm{C}$ for $9 \mathrm{~h}$. After cooling to room temperature, the mixture was acidified with $4 \%$ aq. $\mathrm{HCl}(40 \mathrm{~mL})$ and then extracted with toluene $(6 \times 60 \mathrm{~mL})$. The combined organic solution was washed with brine $(2 \times 60 \mathrm{~mL})$ and dried with anhydrous magnesium sulphate. The solvent was evaporated and the product was purified by column chromatography (toluene/methanol/acetic acid 32/1/1) to yield $2.98 \mathrm{~g}(81 \%)$ of protected acid 2, m.p. 126-130 ${ }^{\circ} \mathrm{C} .{ }^{1} \mathrm{H}$ NMR spectrum $\left(\mathrm{CDCl}_{3}\right): 0.23\left(\mathrm{~s}, 9 \mathrm{H}, \mathrm{C}\left(\mathrm{CH}_{3}\right)_{3}\right), 0.99\left(\mathrm{~s}, 6 \mathrm{H}, 2 \times \mathrm{CH}_{3}\right), 7.07(\mathrm{~m}, 1 \mathrm{H})$, $7.43(\mathrm{~m}, 1 \mathrm{H}), 7.68(\mathrm{~m}, 1 \mathrm{H})$. Elemental analysis: for $\mathrm{C}_{13} \mathrm{H}_{19} \mathrm{ClO}_{3} \mathrm{Si}(286.83)$ : calculated C 54.44, $\mathrm{H}$ 6.68, $\mathrm{Cl} 12.36$; found $\mathrm{C} 54.39, \mathrm{H} 6.90, \mathrm{Cl} 12.22 \%$. 


\section{3-Hydroxy-5-nitrobenzoic acid (10)}

Boron tribromide $(13.8 \mathrm{~mL} ; 153 \mathrm{mmol})$ was added drop wise in an inert argon atmosphere to a solution of acid 6 ( $3.65 \mathrm{~g} ; 18.5 \mathrm{mmol})$ in dry dichloromethane $(40 \mathrm{~mL})$ cooled to $78^{\circ} \mathrm{C}$. The mixture was brought slowly to $0{ }^{\circ} \mathrm{C}$, then stirred at this temperature for $16 \mathrm{~h}$, and decomposed by pouring on ice $(250 \mathrm{~mL})$. The product was extracted with ethyl acetate $(3 \times 80 \mathrm{~mL})$ and the combined organic solution was washed with brine $(80 \mathrm{~mL})$, and dried with anhydrous magnesium sulphate. The solvent was evaporated and the crude product was purified by column chromatography (toluene/methanol/acetic acid 32/1/1) to give rise to $3.06 \mathrm{~g}(90 \%)$ of the corresponding hydroxy acid 10, m.p. $202-206{ }^{\circ} \mathrm{C}$, m.p. $195-197^{\circ} \mathrm{C}$, see reference [37].

\section{3-(tert-Butyldimethylsilyloxy)-5-nitrobenzoic acid (4)}

By the same method as for acid 2, silylation of acid 10 (1.50 g; $8.19 \mathrm{mmol})$ with TBSCl $(1.23 \mathrm{~g}$; $8.19 \mathrm{mmol})$ in the presence of imidazole $(1.12 \mathrm{~g} ; 16.4 \mathrm{mmol})$ yielded $1.45 \mathrm{~g}(60 \%)$ of acid $4, \mathrm{~m} . \mathrm{p} .166-170^{\circ} \mathrm{C}$. ${ }^{1} \mathrm{H}$ NMR spectrum $\left(\mathrm{CDCl}_{3}\right): 0.29\left(\mathrm{~s}, 6 \mathrm{H}, \mathrm{CH}_{3}\right), 1.02\left(\mathrm{~s}, 9 \mathrm{H},\left(\mathrm{CH}_{3}\right)_{3} \mathrm{C}\right), 7.86(\mathrm{~m}, 1 \mathrm{H}, \mathrm{H} 2), 7.90(\mathrm{~m}, 1 \mathrm{H}$, $\mathrm{H} 4), 8.54$ (s, $1 \mathrm{H}, \mathrm{H} 6)$. Elemental analysis: for $\mathrm{C}_{13} \mathrm{H}_{19} \mathrm{NO}_{5} \mathrm{Si}$ (297.39): calculated C 52.51, H 6.44, N 4.71; found C 52.44, H 6.40, N 4.65\%.

\subsection{Synthesis of Intermediates and Target Compounds}

Synthesis of intermediates $\mathbf{1 3 a}-\mathbf{d}, \mathbf{1 5} \mathbf{a}-\mathbf{d}, \mathbf{1 7} \mathbf{a}-\mathbf{d}$, and $\mathbf{1 9 a} \mathbf{a} \mathbf{d}$ and their characterisation was reported in the current paper [36].

\section{4-[4-(Octyloxy)benzoyloxy]phenyl 3-(tert-butyldimethylsilyloxy)-5-chlorobenzoate (14a).}

A mixture of acid $2(0.704 \mathrm{~g} ; 2.45 \mathrm{mmol})$, phenol 11a $(0.680 \mathrm{~g} ; 1.99 \mathrm{mmol})$, DCC $(0.647 \mathrm{~g} ; 3.13 \mathrm{mmol})$, and DMAP $(10 \mathrm{mg})$ in dry dichloromethane $(40 \mathrm{~mL})$ was stirred at room temperature for $2 \mathrm{~h}$ in an inert atmosphere. The deposited solid was filtered and washed with dichloromethane $(20 \mathrm{~mL})$. The filtrate was evaporated and the crude product was purified by column chromatography (hexane/tert-butyl methyl ether 10/1). Yield $0.722 \mathrm{~g}(59 \%)$ of 14a, m.p. $98-99{ }^{\circ} \mathrm{C} .{ }^{1} \mathrm{H}$ NMR spectrum $\left(\mathrm{CDCl}_{3}\right): 0.25(\mathrm{~s}, 9 \mathrm{H}$, $\left.\mathrm{C}\left(\mathrm{CH}_{3}\right)_{3}\right), 0.88\left(\mathrm{~m}, 3 \mathrm{H}, \mathrm{CH}_{3}\right), 1.00\left(\mathrm{~s}, 6 \mathrm{H}, 2 \times \mathrm{CH}_{3}\right), 1.25-1.61\left(\mathrm{~m}, 10 \mathrm{H},\left(\mathrm{CH}_{2}\right)_{5}\right), 1.82\left(\mathrm{~m}, 2 \mathrm{H}, \mathrm{CH}_{2}\right)$, $4.06\left(\mathrm{t}, 2 \mathrm{H}, \mathrm{CH}_{2}, J=6.5\right), 6.97(\mathrm{~d}, 2 \mathrm{H}, J=8.8), 7.10(\mathrm{~m}, 1 \mathrm{H}), 7.25-7.28(\mathrm{~m}, 4 \mathrm{H}), 7.52(\mathrm{~m}, 1 \mathrm{H}), 7.79(\mathrm{~m}$, $1 \mathrm{H}), 8.14(\mathrm{~d}, 2 \mathrm{H}, J=8.8)$. Elemental analysis: for $\mathrm{C}_{34} \mathrm{H}_{43} \mathrm{ClO}_{6} \mathrm{Si}(611.26)$ : calculated C 66.81, $\mathrm{H}$ 7.09, $\mathrm{Cl} 5.80$; found C 66.69, H 7.03, Cl 5.69\%.

In the same way, homologues $14 \mathbf{b}-\mathbf{d}$ have been prepared.

4-[4-(Decyloxy)benzoyloxy]phenyl 3-(tert-butyldimethylsilyloxy)-5-chlorobenzoate (14b). Yield 75\%, m.p. $85-87^{\circ} \mathrm{C}$.

4-[4-(Dodecyloxy)benzoyloxy]phenyl 3-(tert-butyldimethylsilyloxy)-5-chlorobenzoate (14c). Yield 73\%, m.p. $56-58^{\circ} \mathrm{C}$.

4-[4-(Tetradecyloxy)benzoyloxy]phenyl 3-(tert-butyldimethylsilyloxy)-5-chlorobenzoate (14d). Yield 73\%, m.p. $54-56^{\circ} \mathrm{C}$.

By the method shown above, reaction of nitro acid 4 with phenols 11a-d yielded the corresponding intermediates 16a-d.

4-[4-(Octyloxy)benzoyloxy]phenyl 3-(tert-butyldimethylsilyloxy)-5-nitrobenzoate (16a). Yield 59\%, m.p. $100-103{ }^{\circ} \mathrm{C} .{ }^{1} \mathrm{H}$ NMR spectrum $\left(\mathrm{CDCl}_{3}\right): 0.30\left(\mathrm{~s}, 9 \mathrm{H}, \mathrm{C}\left(\mathrm{CH}_{3}\right)_{3}\right), 0.88\left(\mathrm{~m}, 3 \mathrm{H}, \mathrm{CH}_{3}\right), 1.03(\mathrm{~s}, 6 \mathrm{H}$, $\left.2 \times \mathrm{CH}_{3}\right), 1.25-1.54\left(\mathrm{~m}, 10 \mathrm{H},\left(\mathrm{CH}_{2}\right)_{5}\right), 1.81\left(\mathrm{~m}, 2 \mathrm{H}, \mathrm{CH}_{2}\right), 4.05\left(\mathrm{t}, 2 \mathrm{H}, \mathrm{CH}_{2}, J=6.3\right), 6.98(\mathrm{~d}, 2 \mathrm{H}, J=9.1)$, $7.29(\mathrm{~s}, 2 \mathrm{H}), 7.93(\mathrm{~m}, 2 \mathrm{H}), 8.15(\mathrm{~d}, 2 \mathrm{H}, J=9.1), 8.63(\mathrm{~m}, 1 \mathrm{H})$. Elemental analysis: for $\mathrm{C}_{34} \mathrm{H}_{43} \mathrm{NO}_{8} \mathrm{Si}$ (621.81): calculated C 65.68, H 6.97, N 2.25; found C 65.53, H 6.88, N 2.16\%.

4-[4-(Decyloxy)benzoyloxy]phenyl 3-(tert-butyldimethylsilyloxy)-5-nitrobenzoate (16b). Yield 80\%, m.p. $115-119^{\circ} \mathrm{C}$. 
4-[4-(Dodecyloxy)benzoyloxy]phenyl 3-(tert-butyldimethylsilyloxy)-5-nitrobenzoate (16c). Yield 57\%, m.p. $95-99^{\circ} \mathrm{C}$.

4-[4-(Tetradecyloxy)benzoyloxy]phenyl 3-(tert-butyldimethylsilyloxy)-5-nitrobenzoate (16d). Yield 55\%, m.p. $96-101{ }^{\circ} \mathrm{C}$.

4-[4-(Octyloxy)benzoyloxy]phenyl 3-chloro-5-hydroxybenzoate (18a)

A $1 \mathrm{M}$ solution of TBAF in THF $(0.2 \mathrm{~mL}, 0.2 \mathrm{mmol})$ was added drop wise to a solution of $14 \mathrm{a}(487 \mathrm{mg}$; $0.79 \mathrm{mmol})$ in a mixture of THF $(50 \mathrm{~mL})$ and water $(15 \mathrm{~mL})$. The solution was stirred at room temperature for $1 \mathrm{~h}$, diluted with water $(100 \mathrm{~mL})$ and extracted with ethyl acetate $(3 \times 100 \mathrm{~mL})$. The combined extracts were washed with brine $(100 \mathrm{~mL})$ and dried with anhydrous magnesium sulphate. After removing the solvent, the product was purified by column chromatography (hexane/tert-butyl methyl ether 20/1). $0.34 \mathrm{~g}(86 \%)$ of $18 \mathrm{a}$ was isolated, m.p. $169-171{ }^{\circ} \mathrm{C} .{ }^{1} \mathrm{H}$ NMR spectrum $\left(\mathrm{CDCl}_{3}\right): 0.88\left(\mathrm{t}, 3 \mathrm{H}, \mathrm{CH}_{3}\right)$, 1.25-1.54 (m, $\left.10 \mathrm{H},\left(\mathrm{CH}_{2}\right)_{5}\right), 1.81\left(\mathrm{~m}, 2 \mathrm{H}, \mathrm{CH}_{2}\right), 4.05\left(\mathrm{t}, 2 \mathrm{H}, \mathrm{CH}_{2}, J=6.4\right), 5.42(\mathrm{~s}, 1 \mathrm{H}, \mathrm{OH}), 6.97(\mathrm{~d}, 2 \mathrm{H}$, $J=8.8), 7.13(\mathrm{~m}, 1 \mathrm{H}), 7.17-7.31(\mathrm{~m}, 4 \mathrm{H}), 7.54(\mathrm{~m}, 1 \mathrm{H}), 7.77(\mathrm{~m}, 1 \mathrm{H}), 8.14(\mathrm{~d}, 2 \mathrm{H}, J=8.8)$. Elemental analysis: for $\mathrm{C}_{28} \mathrm{H}_{29} \mathrm{ClO}_{6}$ (496.99): calculated C 67.67, $\mathrm{H}$ 5.88, $\mathrm{Cl}$ 7.13; found $\mathrm{C} 67.55, \mathrm{H} 5.76, \mathrm{Cl} 7.03 \%$. In the same manner, deprotection of compounds $\mathbf{1 4 b} \mathbf{b}-\mathbf{d}$ yielded compounds $\mathbf{1 8 b}-\mathbf{d}$.

4-[4-(Decyloxy)benzoyloxy]phenyl 3-chloro-5-hydroxybenzoate (18b). Yield 72\%, m.p. $122-127{ }^{\circ} \mathrm{C}$. 4-[4-(Dodecyloxy)benzoyloxy]phenyl 3-chloro-5-hydroxybenzoate (18c). Yield 84\%, m.p. 96-123 ${ }^{\circ} \mathrm{C}$. 4-[4-(Tetradecyloxy)benzoyloxy]phenyl 3-chloro-5-hydroxybenzoate (18d). Yield 83\%, m.p. 108-119 ${ }^{\circ} \mathrm{C}$.

The 5-nitro substituted intermediates 20a-d have been prepared by deprotection of the compounds 16a-d by the same method.

4-[4-(Octyloxy)benzoyloxy]phenyl 3-hydroxy-5-nitrobenzoate (20a). Yield $80 \%$, m.p. $126-141{ }^{\circ} \mathrm{C} .{ }^{1} \mathrm{H}$ NMR spectrum $\left(\mathrm{CDCl}_{3}\right): 0.88\left(\mathrm{t}, 3 \mathrm{H}, \mathrm{CH}_{3}\right), 1.24-1.52\left(\mathrm{~m}, 10 \mathrm{H},\left(\mathrm{CH}_{2}\right)_{5}\right), 1.83\left(\mathrm{~m}, 2 \mathrm{H}, \mathrm{CH}_{2}\right), 4.05(\mathrm{t}$, $\left.2 \mathrm{H}, \mathrm{CH}_{2}, J=6.4\right), 6.98(\mathrm{~d}, 3 \mathrm{H}, J=9.1), 7.9(\mathrm{~m}, 2 \mathrm{H}), 7.93(\mathrm{~m}, 2 \mathrm{H}), 8.14(\mathrm{~d}, 3 \mathrm{H}, J=8.8), 8.57(\mathrm{~m}, 1 \mathrm{H})$. Elemental analysis: for $\mathrm{C}_{28} \mathrm{H}_{29} \mathrm{NO}_{8}$ (507.55): calculated C 66.26, H 5.76, N 2.76; found C 66.20, H 5.70, N 2.72\%.

4-[4-(Decyloxy)benzoyloxy]phenyl 3-hydroxy-5-nitrobenzoate (20b). Yield 76\%, m.p. $142-144{ }^{\circ} \mathrm{C}$.

4-[4-(Dodecyloxy)benzoyloxy]phenyl 3-hydroxy-5-nitrobenzoate (20c). Yield 90\%, m.p. 132-136 ${ }^{\circ} \mathrm{C}$. 4-[4-(Tetradecyloxy)benzoyloxy]phenyl 3-hydroxy-5-nitrobenzoate (20d). Yield 74\%, m.p. $122-124{ }^{\circ} \mathrm{C}$.

\subsection{Synthesis of Target Compounds}

4-[4-(Octyloxy)benzoyloxy]phenyl 3-fluoro-5-\{4-[4-(octyloxy)benzoyloxy]benzoyloxy\}benzoate (Ia) A mixture of acid 12a $(0.37 \mathrm{~g} ; 1 \mathrm{mmol})$ and oxalyl chloride $(0.43 \mathrm{~mL} ; 5 \mathrm{mmol})$ in dichloromethane $(10 \mathrm{~mL})$ was stirred at room temperature for $12 \mathrm{~h}$ and then evaporated. The crude acid chloride was dissolved in toluene $(30 \mathrm{~mL})$ and added to a solution of $\mathbf{1 7 a}(0.236 \mathrm{~g} ; 0.49 \mathrm{mmol})$ and DMAP $(0.079 \mathrm{~g}$; $0.69 \mathrm{mmol})$ in toluene $(30 \mathrm{~mL})$. The mixture was heated to boiling for $1 \mathrm{~h}$, cooled to room temperature and acidified with $2 \%$ aq. $\mathrm{HCl}(50 \mathrm{~mL})$. The layers were divided and the aqueous phase was extracted with chloroform $(3 \times 50 \mathrm{~mL})$. The collected organic solution was washed with brine $(50 \mathrm{~mL})$ and dried with anhydrous magnesium sulphate. The solvent was evaporated and the crude product was purified by column chromatography (toluene/tert-butyl methyl ether 20/1) and multiple crystallisations from a toluene/acetone mixture. Yield $0.379 \mathrm{~g}(93 \%) .{ }^{1} \mathrm{H}$ NMR spectrum $\left(\mathrm{CDCl}_{3}\right): 0.88\left(\mathrm{~m}, 6 \mathrm{H}, 2 \times \mathrm{CH}_{3}\right)$, 1.25-1.54 (m, $\left.20 \mathrm{H}, 2 \times\left(\mathrm{CH}_{2}\right)_{5}\right), 1.83\left(\mathrm{~m}, 4 \mathrm{H}, 2 \times \mathrm{CH}_{2}\right), 4.05\left(\mathrm{t}, 2 \mathrm{H}, \mathrm{OCH}_{2}, J=6.6\right), 4.06\left(\mathrm{t}, 2 \mathrm{H}, \mathrm{OCH}_{2}\right.$, $J=6.6), 6.98(\mathrm{~m}, 4 \mathrm{H}), 7.22-7.35(\mathrm{~m}, 5 \mathrm{H}), 7.40(\mathrm{~d}, 2 \mathrm{H}, J=8.8), 7.85(\mathrm{~m}, 1 \mathrm{H}), 7.91(\mathrm{~s}, 1 \mathrm{H}), 8.15(\mathrm{~m}, 4 \mathrm{H})$, $8.28(\mathrm{~d}, 2 \mathrm{H}, J=8.5)$. IR (KBr): $3731 \mathrm{~s}, 3627 \mathrm{~s}, 2921 \mathrm{~s}, 2852 \mathrm{~s}, 1734 \mathrm{~s}, 1609 \mathrm{~m}, 1508 \mathrm{v}, 1306 \mathrm{w}, 1260 \mathrm{~s}, 1165 \mathrm{~s}$, 
$1064 \mathrm{~m}, 844 \mathrm{w}, 758 \mathrm{w}$. Elemental analysis: for $\mathrm{C}_{50} \mathrm{H}_{53} \mathrm{FO}_{10}$ (832.97): calculated C 72.10, $\mathrm{H}$ 6.41; found C $72.03, \mathrm{H} 6.33 \%$.

By the same procedure, compounds $\mathbf{I b}-\mathbf{d}$ have been prepared by the reaction of acids $\mathbf{1 2 b} \mathbf{b} \mathbf{d}$ with phenols 17b-d.

4-[4-(Decyloxy)benzoyloxy]phenyl 5-\{4-[4-(decyloxy)benzoyloxy]benzoyloxy\}-3-fluorobenzoate (Ib). Yield 77\%. ${ }^{1} \mathrm{H}$ NMR spectrum $\left(\mathrm{CDCl}_{3}\right): 0.88\left(\mathrm{~m}, 6 \mathrm{H}, 2 \times \mathrm{CH}_{3}\right), 1.24-1.54\left(\mathrm{~m}, 28 \mathrm{H}, 2 \times\left(\mathrm{CH}_{2}\right)_{7}\right), 1.83$ $\left(\mathrm{m}, 4 \mathrm{H}, 2 \times \mathrm{CH}_{2}\right), 4.05\left(\mathrm{t}, 2 \mathrm{H}, \mathrm{OCH}_{2}, J=6.4\right), 4.06\left(\mathrm{t}, 2 \mathrm{H}, \mathrm{OCH}_{2}, J=6.6\right), 6.98(\mathrm{~m}, 4 \mathrm{H}), 7.12-7.35(\mathrm{~m}$, $5 \mathrm{H}), 7.40(\mathrm{~d}, 2 \mathrm{H}, J=8.5), 7.84(\mathrm{~m}, 1 \mathrm{H}), 7.89(\mathrm{~s}, 1 \mathrm{H}), 8.15(\mathrm{~m}, 4 \mathrm{H}), 8.28(\mathrm{~d}, 2 \mathrm{H}, J=8.8)$. IR (KBr): 3729 s, 3614 s, 2920 s, 2853 s, 1736 s, 1607 m, 1509 v, 1306 w, 1261 s, 1208 s, 1167 s, 1063 m, 879 w, 758 w. Elemental analysis: for $\mathrm{C}_{54} \mathrm{H}_{61} \mathrm{FO}_{10}$ (889.08): calculated $\mathrm{C} 72.95, \mathrm{H}$ 6.92; found $\mathrm{C} 72.86, \mathrm{H} 6.85 \%$.

4-[4-(Dodecyloxy)benzoyloxy]phenyl 5-\{4-[4-(dodecyloxy)benzoyloxy]benzoyloxy\}-3-fluorobenzoate (Ic). Yield 78\%. ${ }^{1} \mathrm{H}$ NMR spectrum $\left(\mathrm{CDCl}_{3}\right): 0.88\left(\mathrm{~m}, 6 \mathrm{H}, 2 \times \mathrm{CH}_{3}\right), 1.21-1.56(\mathrm{~m}, 36 \mathrm{H}, 2 \times$ $\left.\left(\mathrm{CH}_{2}\right)_{9}\right), 1.83\left(\mathrm{~m}, 4 \mathrm{H}, 2 \times \mathrm{CH}_{2}\right), 4.05\left(\mathrm{t}, 2 \mathrm{H}, \mathrm{OCH}_{2}, J=6.6\right), 4.06\left(\mathrm{t}, 2 \mathrm{H}, \mathrm{OCH}_{2}, J=6.6\right), 6.98(\mathrm{~m}, 4 \mathrm{H})$, 7.24-7.35 (m, $5 \mathrm{H}), 7.40(\mathrm{~d}, 2 \mathrm{H}, J=8.8), 7.83(\mathrm{~m}, 1 \mathrm{H}, \mathrm{H} 2), 7.90(\mathrm{~s}, 1 \mathrm{H}, \mathrm{H} 6), 8.15(\mathrm{~m}, 4 \mathrm{H}), 8.29(\mathrm{~d}, 2 \mathrm{H}$, $J=8.8)$. IR (KBr): $3741 \mathrm{~s}, 3636 \mathrm{~s}, 2918 \mathrm{~s}, 2852 \mathrm{~s}, 1736 \mathrm{~s}, 1606 \mathrm{~m}, 1510 \mathrm{v}, 1470 \mathrm{w}, 1306 \mathrm{w}, 1262 \mathrm{~s}, 1208 \mathrm{~m}$, 1167 s, $1132 \mathrm{~m}, 1063 \mathrm{~m}, 842 \mathrm{w}, 758 \mathrm{w}$. Elemental analysis: for $\mathrm{C}_{58} \mathrm{H}_{69} \mathrm{FO}_{10}$ (945.19): calculated C 73.70, H 7.36; found C 73.59, H 7.32\%.

4-[4-(Tetradecyloxy)benzoyloxy]phenyl 3-fluoro-5-\{4-[4-(tetradecyloxy)benzoyloxy]benzoyloxy\}benzoate (Id). Yield 90\%. ${ }^{1} \mathrm{H}$ NMR spectrum $\left(\mathrm{CDCl}_{3}\right): 0.88\left(\mathrm{~m}, 6 \mathrm{H}, 2 \times \mathrm{CH}_{3}\right), 1.20-1.54\left(\mathrm{~m}, 44 \mathrm{H}, 2 \times\left(\mathrm{CH}_{2}\right){ }_{11}\right)$, $1.83\left(\mathrm{~m}, 4 \mathrm{H}, 2 \times \mathrm{CH}_{2}\right), 4.05\left(\mathrm{t}, 2 \mathrm{H}, \mathrm{OCH}_{2}, J=6.6\right), 4.06\left(\mathrm{t}, 2 \mathrm{H}, \mathrm{OCH}_{2}, J=6.6\right), 6.99(\mathrm{~m}, 4 \mathrm{H}), 7.22-7.34$ $(\mathrm{m}, 5 \mathrm{H}), 7.40(\mathrm{~d}, 2 \mathrm{H}, J=8.5), 7.83(\mathrm{~m}, 1 \mathrm{H}, \mathrm{H} 2), 7.87(\mathrm{~s}, 1 \mathrm{H}, \mathrm{H} 6), 8.16(\mathrm{~m}, 4 \mathrm{H}), 8.29(\mathrm{~d}, 2 \mathrm{H}, J=9.1)$. IR (KBr): 3742 w, 2918 s, 2851 s, 1735 s, 1606 m, 1510 v, 1470 w, 1447 w, 1321 w, 1259 s, 1209 m, 1168 s, $1132 \mathrm{~m}, 1063 \mathrm{~m}, 843 \mathrm{w}, 758 \mathrm{w}$. Elemental analysis: for $\mathrm{C}_{62} \mathrm{H}_{77} \mathrm{FO}_{10}$ (1001.30): calculated C 74.37, H 7.75; found C 74.26, H 7.66\%.

The procedure for synthesis Ia was further utilized for the synthesis of compounds of series IIa-d by the reaction of acids 12a-d with intermediates $18 a-d$, series IIIa-d by the reaction of acids $12 a-d$ with intermediates $\mathbf{1 9} \mathbf{a}-\mathbf{d}$, and compounds IVa-d by the reaction of acids $12 \mathbf{a}-\mathbf{d}$ with intermediates 20a-d, respectively.

4-[4-(Octyloxy)benzoyloxy]phenyl 3-chloro-5-\{4-[4-(octyloxy)benzoyloxy]benzoyloxy\}benzoate (IIa). Yield 97\%. ${ }^{1} \mathrm{H}$ NMR spectrum $\left(\mathrm{CDCl}_{3}\right): 0.88\left(\mathrm{~m}, 6 \mathrm{H}, 2 \times \mathrm{CH}_{3}\right), 1.22-1.54\left(\mathrm{~m}, 20 \mathrm{H}, 2 \times\left(\mathrm{CH}_{2}\right)_{5}\right), 1.81(\mathrm{~m}$, $\left.4 \mathrm{H}, 2 \times \mathrm{CH}_{2}\right), 4.05\left(\mathrm{t}, 2 \mathrm{H}, \mathrm{CH}_{2}, J=6.3\right), 4.06\left(\mathrm{t}, 2 \mathrm{H}, \mathrm{CH}_{2}, J=6.4\right), 6.98(\mathrm{~m}, 4 \mathrm{H}), 7.27(\mathrm{~s}, 4 \mathrm{H}), 7.40(\mathrm{~d}$, $2 \mathrm{H}, J=8.8), 7.57(\mathrm{~m}, 1 \mathrm{H}, J=2.0), 7.98(\mathrm{~m}, 1 \mathrm{H}, J=1.2), 8.15(\mathrm{~m}, 5 \mathrm{H}), 8.28(\mathrm{~d}, 2 \mathrm{H}, J=8.8)$. Elemental analysis: for $\mathrm{C}_{50} \mathrm{H}_{53} \mathrm{ClO}_{10}$ (849.43): calculated C 70.70, $\mathrm{H} 6.29$; found $\mathrm{C} 70.58, \mathrm{H} 6.30 \%$.

4-[4-(Decyloxy)benzoyloxy]phenyl 3-chloro-5-\{4-[4-(decyloxy)benzoyloxy]benzoyloxy\}benzoate (IIb). Yield 92\%. ${ }^{1} \mathrm{H}$ NMR spectrum $\left(\mathrm{CDCl}_{3}\right): 0.88\left(\mathrm{~m}, 6 \mathrm{H}, 2 \times \mathrm{CH}_{3}\right), 1.25-1.55\left(\mathrm{~m}, 28 \mathrm{H}, 2 \times\left(\mathrm{CH}_{2}\right)_{7}\right)$, $1.81\left(\mathrm{~m}, 4 \mathrm{H}, 2 \times \mathrm{CH}_{2}\right), 4.05\left(\mathrm{t}, 2 \mathrm{H}, \mathrm{CH}_{2}, J=6.3\right), 4.06\left(\mathrm{t}, 2 \mathrm{H}, \mathrm{CH}_{2}, J=6.3\right), 6.98(\mathrm{~m}, 4 \mathrm{H}), 7.28(\mathrm{~s}, 4 \mathrm{H})$, $7.40(\mathrm{~d}, 2 \mathrm{H}, J=8.8), 7.57(\mathrm{~m}, 1 \mathrm{H}), 7.98(\mathrm{~m}, 1 \mathrm{H}), 8.14(\mathrm{~m}, 5 \mathrm{H}), 8.28(\mathrm{~d}, 2 \mathrm{H}, J=8.8)$. Elemental analysis: for $\mathrm{C}_{54} \mathrm{H}_{61} \mathrm{ClO}_{10}$ (905.54): calculated C 71.63, $\mathrm{H}$ 6.79; found C 71.55, $\mathrm{H} 6.71 \%$.

4-[4-(Dodecyloxy)benzoyloxy]phenyl 3-chloro-5-\{4-[4-(dodecyloxy)benzoyloxy]benzoyloxy\}benzoate (IIc). Yield 92\%. ${ }^{1} \mathrm{H}$ NMR spectrum $\left(\mathrm{CDCl}_{3}\right): 0.88\left(\mathrm{~m}, 6 \mathrm{H}, 2 \times \mathrm{CH}_{3}\right), 1.27-1.54\left(\mathrm{~m}, 36 \mathrm{H}, 2 \times\left(\mathrm{CH}_{2}\right)_{9}\right)$, $1.81\left(\mathrm{~m}, 4 \mathrm{H}, 2 \times \mathrm{CH}_{2}\right), 4.05\left(\mathrm{t}, 2 \mathrm{H}, \mathrm{CH}_{2}, J=6.3\right), 4.06\left(\mathrm{t}, 2 \mathrm{H}, \mathrm{CH}_{2}, J=6.4\right), 6.98(\mathrm{~m}, 4 \mathrm{H}), 7.28(\mathrm{~s}, 4 \mathrm{H})$, $7.40(\mathrm{~d}, 2 \mathrm{H}, J=8.8), 7.57(\mathrm{~m}, 1 \mathrm{H}), 7.98(\mathrm{~m}, 1 \mathrm{H}), 8.14(\mathrm{~m}, 5 \mathrm{H}), 8.28(\mathrm{~d}, 2 \mathrm{H}, J=8.8)$. Elemental analysis: for $\mathrm{C}_{58} \mathrm{H}_{69} \mathrm{ClO}_{10}$ (961.64): calculated C 72.44, $\mathrm{H}$ 7.23; found C 72.40, $\mathrm{H} 7.19 \%$.

4-[4-(Tetradecyloxy)benzoyloxy]phenyl 3-chloro-5-\{4-[4-(tetradecyloxy)benzoyloxy]benzoyloxy\}benzoate (IId). Yield 98\%. ${ }^{1} \mathrm{H}$ NMR spectrum $\left(\mathrm{CDCl}_{3}\right): 0.88\left(\mathrm{~m}, 6 \mathrm{H}, 2 \times \mathrm{CH}_{3}\right) 1.26-1.56\left(\mathrm{~m}, 44 \mathrm{H}, 2 \times\left(\mathrm{CH}_{2}\right)_{11}\right)$, $1.81\left(\mathrm{~m}, 4 \mathrm{H}, 2 \times \mathrm{CH}_{2}\right), 4.05\left(\mathrm{t}, 2 \mathrm{H}, \mathrm{CH}_{2}, J=6.3\right), 4.06\left(\mathrm{t}, 2 \mathrm{H}, \mathrm{CH}_{2}, J=6.4\right), 6.98(\mathrm{~m}, 4 \mathrm{H}), 7.28(\mathrm{~s}, 4 \mathrm{H})$, 
$7.40(\mathrm{~d}, 2 \mathrm{H}, J=8.8), 7.57(\mathrm{~m}, 1 \mathrm{H}), 7.98(\mathrm{~m}, 1 \mathrm{H}), 8.14(\mathrm{~m}, 5 \mathrm{H}), 8.28(\mathrm{~d}, 2 \mathrm{H}, J=9.1)$. Elemental analysis: for $\mathrm{C}_{62} \mathrm{H}_{77} \mathrm{ClO}_{10}$ (1017.75): calculated C 73.14, $\mathrm{H}$ 7.63; found C 73.01, $\mathrm{H} 7.56 \%$.

4-[4-(Octyloxy)benzoyloxy]phenyl 3-methyl-5-\{4-[4-(octyloxy)benzoyloxy]benzoyloxy\}benzoate (IIIa). Yield 75\%. ${ }^{1} \mathrm{H}$ NMR spectrum $\left(\mathrm{CDCl}_{3}\right): 0.88\left(\mathrm{~m}, 6 \mathrm{H}, 2 \times \mathrm{CH}_{3}\right), 1.22-1.54\left(\mathrm{~m}, 20 \mathrm{H}, 2 \times\left(\mathrm{CH}_{2}\right)_{5}\right), 1.81(\mathrm{~m}$, $\left.4 \mathrm{H}, 2 \times \mathrm{CH}_{2}\right), 2.50\left(\mathrm{~s}, 3 \mathrm{H}, \mathrm{CH}_{3}\right), 4.05\left(\mathrm{t}, 2 \mathrm{H}, \mathrm{OCH}_{2}, J=6.3\right), 4.06\left(\mathrm{t}, 2 \mathrm{H}, \mathrm{OCH}_{2}, J=6.4\right), 6.98(\mathrm{~m}, 4 \mathrm{H}), 7.27$ $(\mathrm{s}, 4 \mathrm{H}), 7.34(\mathrm{~s}, 1 \mathrm{H}), 7.40(\mathrm{~d}, 2 \mathrm{H}, J=8.8), 7.87(\mathrm{~s}, 1 \mathrm{H}), 7.95(\mathrm{~s}, 1 \mathrm{H}), 8.15(\mathrm{~m}, 4 \mathrm{H}), 8.28(\mathrm{~d}, 2 \mathrm{H}, J=8.8)$. IR (KBr): 3729 w, 3625 w, 2923 s, 2856 s, 1735 s, 1606 m, 1509 v, 1306 w, 1259 s, 1164 s, 1066 m, 844 w, 757 w. Elemental analysis: for $\mathrm{C}_{51} \mathrm{H}_{56} \mathrm{O}_{10}$ (829.01): calculated C 73.89, $\mathrm{H}$ 6.81; found C 73.77, H 6.69\%.

4-[4-(Decyloxy)benzoyloxy]phenyl 5-\{4-[4-(decyloxy)benzoyloxy]benzoyloxy\}-3-methylbenzoate (IIIb). Yield 75\%. ${ }^{1} \mathrm{H}$ NMR spectrum $\left(\mathrm{CDCl}_{3}\right): 0.88\left(\mathrm{~m}, 6 \mathrm{H}, 2 \times \mathrm{CH}_{3}\right), 1.25-1.55\left(\mathrm{~m}, 28 \mathrm{H}, 2 \times\left(\mathrm{CH}_{2}\right)_{7}\right), 1.81(\mathrm{~m}$, $\left.4 \mathrm{H}, 2 \times \mathrm{CH}_{2}\right), 2.49\left(\mathrm{~s}, 3 \mathrm{H}, \mathrm{CH}_{3}\right), 4.05\left(\mathrm{t}, 2 \mathrm{H}, \mathrm{OCH}_{2}, J=6.3\right), 4.06\left(\mathrm{t}, 2 \mathrm{H}, \mathrm{OCH}_{2}, J=6.3\right), 6.98(\mathrm{~m}, 4 \mathrm{H}), 7.28$ $(\mathrm{s}, 4 \mathrm{H}), 7.34(\mathrm{~s}, 1 \mathrm{H}), 7.41(\mathrm{~d}, 2 \mathrm{H}, J=8.8), 7.86(\mathrm{~s}, 1 \mathrm{H}), 7.94(\mathrm{~s}, 1 \mathrm{H}), 8.15(\mathrm{~m}, 4 \mathrm{H}), 8.28(\mathrm{~d}, 2 \mathrm{H}, J=8.8)$. IR (KBr): 3728 w, 3625 w, 2923 s, 2854 s, 1735 s, 1606 m, 1509 v, 1308 w, 1259 s, 1169 s, 1069 m, 844 w, 759 w. Elemental analysis: for $\mathrm{C}_{55} \mathrm{H}_{64} \mathrm{O}_{10}$ (885.12): calculated C 74.64, $\mathrm{H}$ 7.21; found C 74.44, $\mathrm{H}$ 7.10\%.

4-[4-(Dodecyloxy)benzoyloxy]phenyl 5-\{4-[4-(dodecyloxy)benzoyloxy]benzoyloxy\}-3-methylbenzoate (IIIc). Yield 58\%. ${ }^{1} \mathrm{H}$ NMR spectrum $\left(\mathrm{CDCl}_{3}\right): 0.88\left(\mathrm{~m}, 6 \mathrm{H}, 2 \times \mathrm{CH}_{3}\right), 1.27-1.54\left(\mathrm{~m}, 36 \mathrm{H}, 2 \times\left(\mathrm{CH}_{2}\right)_{9}\right)$, $1.81\left(\mathrm{~m}, 4 \mathrm{H}, 2 \times \mathrm{CH}_{2}\right), 2.50\left(\mathrm{~s}, 3 \mathrm{H}, \mathrm{CH}_{3}\right), 4.05\left(\mathrm{t}, 2 \mathrm{H}, \mathrm{OCH}_{2}, J=6.3\right), 4.06\left(\mathrm{t}, 2 \mathrm{H}, \mathrm{OCH}_{2}, J=6.4\right), 6.98$ $(\mathrm{m}, 4 \mathrm{H}), 7.29(\mathrm{~s}, 4 \mathrm{H}), 7.34(\mathrm{~s}, 1 \mathrm{H}), 7.40(\mathrm{~d}, 2 \mathrm{H}, J=8.8), 7.88(\mathrm{~s}, 1 \mathrm{H}), 7.95(\mathrm{~s}, 1 \mathrm{H}), 8.14(\mathrm{~m}, 4 \mathrm{H}), 8.28(\mathrm{~d}$, $2 \mathrm{H}, J=8.8)$. IR (KBr): $3729 \mathrm{w}, 3627 \mathrm{w}, 2922 \mathrm{~s}, 2853 \mathrm{~m}, 1735 \mathrm{~s}, 1605 \mathrm{~m}, 1509 \mathrm{v}, 1309 \mathrm{w}, 1259 \mathrm{~s}, 1167 \mathrm{~s}$, $1068 \mathrm{~m}, 845 \mathrm{w}, 759 \mathrm{w}$. Elemental analysis: for $\mathrm{C}_{59} \mathrm{H}_{72} \mathrm{O}_{10}$ (941.23): calculated C 75.29, $\mathrm{H} 7.71$; found C 75.20, H $7.56 \%$.

4-[4-(Tetradecyloxy)benzoyloxy]phenyl 3-methyl-5-\{4-[4-(tetradecyloxy)benzoyloxy]benzoyloxy\}benzoate (IIId). Yield 7\%. ${ }^{1} \mathrm{H}$ NMR spectrum $\left(\mathrm{CDCl}_{3}\right): 0.88\left(\mathrm{~m}, 6 \mathrm{H}, 2 \times \mathrm{CH}_{3}\right), 1.26-1.56\left(\mathrm{~m}, 44 \mathrm{H}, 2 \times\left(\mathrm{CH}_{2}\right)_{11}\right)$, $1.81\left(\mathrm{~m}, 4 \mathrm{H}, 2 \times \mathrm{CH}_{2}\right), 2.50\left(\mathrm{~s}, 3 \mathrm{H}, \mathrm{CH}_{3}\right), 4.05\left(\mathrm{t}, 2 \mathrm{H}, \mathrm{OCH}_{2}, J=6.3\right), 4.06\left(\mathrm{t}, 2 \mathrm{H}, \mathrm{OCH}_{2}, J=6.4\right), 6.98$ $(\mathrm{m}, 4 \mathrm{H}), 7.28(\mathrm{~s}, 4 \mathrm{H}), 7.34(\mathrm{~s}, 1 \mathrm{H}), 7.40(\mathrm{~d}, 2 \mathrm{H}, J=8.8), 7.87(\mathrm{~s}, 1 \mathrm{H}), 7.95(\mathrm{~s}, 1 \mathrm{H}), 8.15(\mathrm{~m}, 4 \mathrm{H}), 8.28(\mathrm{~d}$, $2 \mathrm{H}, J=9.1)$. IR (KBr): $3729 \mathrm{w}, 3636 \mathrm{w}, 2929 \mathrm{~s}, 2852 \mathrm{~m}, 1736 \mathrm{~s}, 1606 \mathrm{~m}, 1508 \mathrm{v}, 1308 \mathrm{w}, 1257 \mathrm{~s}, 1167 \mathrm{~s}$, $1068 \mathrm{~m}, 845 \mathrm{w}, 758 \mathrm{w}$. Elemental analysis: for $\mathrm{C}_{63} \mathrm{H}_{80} \mathrm{O}_{10}$ (997.33): calculated C 75.87, $\mathrm{H}$ 8.09; found C 75.69, H 7.97\%.

4-[4-(Octyloxy)benzoyloxy]phenyl 3-nitro-5-\{4-[4-(octyloxy)benzoyloxy]benzoyloxy\}benzoate (IVa). Yield 97\%. ${ }^{1} \mathrm{H}$ NMR spectrum $\left(\mathrm{CDCl}_{3}\right)$ : $0.88\left(\mathrm{t}, 6 \mathrm{H}, 2 \times \mathrm{CH}_{3}\right), 1.25-1.40\left(\mathrm{~m}, 20 \mathrm{H}, 2 \times\left(\mathrm{CH}_{2}\right)_{5}\right), 1.83(\mathrm{~m}$, $\left.4 \mathrm{H}, 2 \times \mathrm{CH}_{2}\right), 4.05\left(\mathrm{t}, 2 \mathrm{H}, \mathrm{CH}_{2}, J=6.5\right), 4.06\left(\mathrm{t}, 2 \mathrm{H}, \mathrm{CH}_{2}, J=6.2\right), 6.98(\mathrm{~d}, 2 \mathrm{H}, J=8.8), 6.99(\mathrm{~d}, 2 \mathrm{H}$, $J=8.8), 7.30(\mathrm{~s}, 4 \mathrm{H}), 7.44(\mathrm{~d}, 2 \mathrm{H}, J=8.8), 8.14(\mathrm{~d}, 2 \mathrm{H}, J=8.8), 8.16(\mathrm{~d}, 2 \mathrm{H}, J=8.8), 8.31(\mathrm{~d}, 2 \mathrm{H}, J=8.8)$, 8.42 (m, 2 H), 8.94 (m, 1 H). IR (ATR): $3110 \mathrm{w}, 2918 \mathrm{~s}, 2855 \mathrm{~s}, 1731 \mathrm{~s}, 1600 \mathrm{~s}, 1543 \mathrm{~m}, 1504 \mathrm{~m}, 1471 \mathrm{w}$, $1252 \mathrm{~s}, 1160 \mathrm{~s}, 1072 \mathrm{~s}, 910 \mathrm{~m}, 839 \mathrm{~m}, 792 \mathrm{~m}$. Elemental analysis: for $\mathrm{C}_{50} \mathrm{H}_{53} \mathrm{NO}_{12}$ (859.98): calculated C 69.83, H 6.21, N 1.63; found C 69.68, H 6.11, N 1.50\%.

4-[4-(Decyloxy)benzoyloxy]phenyl 5-\{4-[4-(decyloxy)benzoyloxy]benzoyloxy\}-3-nitrobenzoate (IVb). Yield $84 \% .{ }^{1} \mathrm{H}$ NMR spectrum $\left(\mathrm{CDCl}_{3}\right): 0.88\left(\mathrm{~m}, 6 \mathrm{H}, 2 \times \mathrm{CH}_{3}\right), 1.25-1.45\left(\mathrm{~m}, 28 \mathrm{H}, 2 \times\left(\mathrm{CH}_{2}\right)_{7}\right), 1.83$ $\left(\mathrm{m}, 4 \mathrm{H}, 2 \times \mathrm{CH}_{2}\right), 4.05\left(\mathrm{t}, 2 \mathrm{H}, \mathrm{CH}_{2}, J=6.4\right), 4.06\left(\mathrm{t}, 2 \mathrm{H}, \mathrm{CH}_{2}, J=6.6\right), 6.98(\mathrm{~d}, 2 \mathrm{H}, J=8.8), 6.99(\mathrm{~d}, 2 \mathrm{H}$, $J=8.8), 7.30(\mathrm{~s}, 4 \mathrm{H}), 7.43(\mathrm{~d}, 2 \mathrm{H}, J=8.8), 8.14(\mathrm{~d}, 2 \mathrm{H}, J=8.8), 8.16(\mathrm{~d}, 2 \mathrm{H}, J=8.8), 8.30(\mathrm{~d}, 2 \mathrm{H}, J=8.8)$, 8.42 (m, 2 H), 8.97 (m, 1 H). IR (ATR): 3115 w, 2916 s, 2851 m, 1726 s, 1604 m, 1543 m, 1509 m, 1468 w, $1250 \mathrm{~s}, 1199 \mathrm{~m}, 1160 \mathrm{~s}, 1062 \mathrm{~s}, 1017 \mathrm{~m}, 841 \mathrm{~m}, 757 \mathrm{~m}$. Elemental analysis: for $\mathrm{C}_{54} \mathrm{H}_{61} \mathrm{NO}_{12}$ (916.09): calculated C 70.80, H 6.71, N 1.53; found C 70.69, H 6.59, N 1.44\%.

4-[4-(Dodecyloxy)benzoyloxy]phenyl 5-\{4-[4-(dodecyloxy)benzoyloxy]benzoyloxy\}-3-nitrobenzoate (IVc). Yield 95\%. ${ }^{1} \mathrm{H}$ NMR spectrum $\left(\mathrm{CDCl}_{3}\right): 0.88\left(\mathrm{t}, 6 \mathrm{H}, 2 \times \mathrm{CH}_{3}\right), 1.22-1.40\left(\mathrm{~m}, 36 \mathrm{H}, 2 \times\left(\mathrm{CH}_{2}\right)_{9}\right)$, $1.83\left(\mathrm{~m}, 4 \mathrm{H}, \mathrm{CH}_{2}\right), 4.05\left(\mathrm{t}, 2 \mathrm{H}, \mathrm{CH}_{2}, J=6.4\right), 4.06\left(\mathrm{t}, 2 \mathrm{H}, \mathrm{CH}_{2}, J=6.6\right), 6.98(\mathrm{~d}, 2 \mathrm{H}, J=8.8), 6.99(\mathrm{~d}, 2 \mathrm{H}$, $J=8.8), 7.31(\mathrm{~s}, 4 \mathrm{H}), 7.43(\mathrm{~d}, 2 \mathrm{H}, J=8.8), 8.14(\mathrm{~d}, 2 \mathrm{H}, J=8.8), 8.16(\mathrm{~d}, 2 \mathrm{H}, J=8.8), 8.31(\mathrm{~d}, 2 \mathrm{H}, J=8.7)$, 8.42 (m, 2 H), 8.97 (m, 1 H). IR (ATR): 3125 w, 3110 w, 2914 s, 2850 s, 1734 s, 1725 s, 1606 m, 1543 m, 
1509 m, 1470 w, 1251 s, 1199 m, 1167 s, 1065 s, 1018 m, 875 m, 846 m, 750 m. Elemental analysis: for $\mathrm{C}_{58} \mathrm{H}_{69} \mathrm{NO}_{12}$ (972.20): calculated C 71.66, H 7.15, N 1.44; found C 71.60, H 7.04, N 1.36\%.

4-[4-(Tetradecyloxy)benzoyloxy]phenyl 3-nitro-5-\{4-[4-(tetradecyloxy)benzoyloxy]benzoyloxy\}benzoate (IVd). Yield 91\%. ${ }^{1} \mathrm{H}$ NMR spectrum $\left(\mathrm{CDCl}_{3}\right): 0.88\left(\mathrm{~m}, 6 \mathrm{H}, 2 \times \mathrm{CH}_{3}\right), 1.26-1.50\left(\mathrm{~m}, 44 \mathrm{H}, 2 \times\left(\mathrm{CH}_{2}\right)_{11}\right)$, $1.82\left(\mathrm{~m}, 4 \mathrm{H}, 2 \times \mathrm{CH}_{2}\right) 4.05\left(\mathrm{t}, 2 \mathrm{H}, \mathrm{CH}_{2}, J=6.6\right), 4.06\left(\mathrm{t}, 2 \mathrm{H}, \mathrm{CH}_{2}, J=6.5\right), 6.98(\mathrm{~d}, 2 \mathrm{H}, J=8.8), 6.99$ $(\mathrm{d}, 2 \mathrm{H}, J=8.8), 7.30(\mathrm{~s}, 2 \mathrm{H}), 7.42(\mathrm{~d}, 2 \mathrm{H}, J=8.8 \mathrm{~Hz}), 8.14(\mathrm{~d}, 2 \mathrm{H}, J=8.8), 8.16(\mathrm{~d}, 2 \mathrm{H}, J=8.8), 8.30$ (d, $2 \mathrm{H}, J=8.8), 8.42(\mathrm{~m}, 2 \mathrm{H}), 8.97(\mathrm{~m}, 1 \mathrm{H})$. IR (ATR): $3117 \mathrm{w}, 2916 \mathrm{~s}, 2851 \mathrm{~s}, 1736 \mathrm{~s}, 1726 \mathrm{~s}, 1604 \mathrm{~m}$, 1543 m, 1508 m, 1475 w, 1249 s, 1198 m, 1166 s, 1064 s, 1016 m, 875 m, 755 m. Elemental analysis: for $\mathrm{C}_{62} \mathrm{H}_{77} \mathrm{NO}_{12}$ (1028.30): calculated C 72.42, H 7.55, N 1.36; found C 72.31, H 7.52, N 1.27\%.

\subsection{Characterization}

The structure of intermediates and target materials were confirmed by ${ }^{1} \mathrm{H}$ NMR spectroscopy (Varian Gemini $300 \mathrm{HC}$ instrument, Varian, Palo Alto, CA, USA), deuteriochloroform and acetone- $d_{6}$ were used and signals of the solvents served as internal standards. Chemical shifts are given in ppm and $J$ values in Hz. Infrared (IR) spectra were acquired on Thermo Scientific Nicolet FT-IR spectrometer in KBr discs or on Bruker ALPHA FT-IR (Bruker, Santa Barbara, CA USA) using attenuated total reflectance (ATR) technique. Elemental analyses were carried out using Elementar vario EL III instrument (Elementar Analysensysteme GmbH, Langenselbold, Germany). Chemical purity of target materials was verified by high-performance liquid chromatography analysis on a Luna Silica column $(150 \times 4.6 \mathrm{ID}, 5 \mu)$ (Phenomenex, Aschaffenburg, Germany) and found to be $\geq 99.8 \%$. Column chromatography was performed using Merck Kieselgel $60(60-100 \mu \mathrm{m})$ (Merck, Darmstadt, Germany). The experimental part summarizes syntheses and spectral data of the selected homologues and all target compounds of series I-IVa-d. In the case of intermediates, ${ }^{1} \mathrm{H}$ NMR characterization is reported for the homologue with the shortest terminal alkyl chain only. Other homologues differ only in the integral value of methylene units in the side chains.

\subsection{Equipment and Set-up for Studies of Mesomorphic Property}

Differential scanning calorimetry (DSC) measurements were carried out on a Perkin-Elmer 7 Pyris calorimeter (PerkinElmer, Shelton, CT, USA). Phase transition temperatures and corresponding enthalpies were determined from the second heating and cooling runs, which were taken at a rate of $10 \mathrm{~K} / \mathrm{min}$. A small amount of compound (2-5 mg) was hermetically closed in aluminum measuring pans and inserted into the calorimeter working chamber. During measurements, a nitrogen atmosphere was applied for better temperature stabilization. The calorimeter was calibrated on extrapolated onset of the melting points of water, indium and zinc.

Electro-optical properties were studied using transparent sample cells fabricated from glass with transparent ITO electrodes $\left(5 \times 5 \mathrm{~mm}^{2}\right)$. The glass plates were separated by mylar sheets to establish the cell thickness. The studied materials were heated to the isotropic phase (Iso) to fill the cells by capillary action. A Nikon Eclipse polarising optical microscope (Nikon, Tokyo, Japan) was used to observe textures and their changes with temperature. Another type of cell (one-free-surface sample) was prepared when we removed the upper glass from a cell without any surface treatment. Temperature stabilization within $\pm 0.1 \mathrm{~K}$ and temperature changes were achieved by the Linkam LTS E350 heating/cooling stage (Linkam, Tadworth, UK) with temperature programmer.

Electric field was applied using driving voltage from generator Philips PM 5191 (Philips Eindhoven, Netherlands), the signal was amplified to reach the maximum amplitude of about $\pm 120 \mathrm{~V}$. A Tektronix DPO4034 digital oscilloscope (Tektronix, Beaverton, OR, USA) was utilized to obtain information about the switching current profile vs. time. Dielectric spectroscopy was measured by Solartron impedance analyser (Solartron Analytical, Farnborough, UK) to establish complex permittivity, $\varepsilon^{*}(\mathrm{f})$, 
in frequency range $10 \mathrm{~Hz}$ to $10 \mathrm{MHz}$. The real, $\varepsilon^{\prime}$, and imaginary, $\varepsilon^{\prime \prime}$, parts of permittivity were fitted to the Cole-Cole formula:

$$
\varepsilon^{*}-\varepsilon_{\infty}=\frac{\Delta \varepsilon}{1+\left(i f / f_{r}\right)^{(1-\alpha)}}-i\left(\frac{\sigma}{2 \pi \varepsilon_{0} f^{n}}+A f^{m}\right)
$$

where $f_{r}$ is the relaxation frequency, $\Delta \varepsilon$ is the dielectric strength, $\alpha$ is the distribution parameter of relaxation, $\varepsilon_{0}$ is the permittivity of vacuum, $\varepsilon_{\infty}$ is the high frequency permittivity and $n, m$, A are the parameters of fitting. We obtained $f_{r}$ and $\Delta \varepsilon$ values to assess dynamic properties and the relaxation process in studied compounds.

$\mathrm{X}$-ray diffraction measurements were performed using Bruker GADDS system ( $\mathrm{CuK} \alpha$ radiation, point beam collimator, Vantec 2000 area detector) working in the reflexion mode. The set-up was equipped with a modified Linkam heating stage with the temperature stability of $0.1 \mathrm{~K}$. Partially oriented samples for experiments were prepared as films on a heated surface.

\section{Results}

\subsection{Mesomorphic Properties and Electro-Optical Behaviour}

All compounds were studied by a calorimetric method; DSC measurements were performed at the heating and cooling runs. We analyzed the measured data and the phase transition temperatures and corresponding enthalpy values (Table 1). The transition peaks observed in DSC plots were sharp and revealed big enthalpy values associated with phase transitions, for demonstration see Figure 1 with thermographs for two selected compounds from the series I. We observed textures under a polarizing microscope to assess mesomorphic behavior. We found that only series I showed mesogenic properties. Namely, for shorter homologues $\mathbf{I a}$ and $\mathbf{I b}$ a columnar $\mathbf{B}_{1}$-type of a mesophase was established from textural features. For illustration, Figure 2a shows the planar texture for Ia with a texture typical for a columnar $\mathrm{B}_{1}$ phase. We observed colored domain-like textures, which did not respond to an applied electric field. For compounds Ic and Id from series I, a SmCP phase appeared with typical textures and specific electro-optical properties. For samples with one free surface, a schlieren texture was found (Figure 2b). In the planar textures without the electric field, we could distinguish a fine structure of stripes. Nevertheless, the averaged extinction without the electric field was oriented along polarizer's direction. Under the applied electric field, the color of the observed fan-shaped texture slightly changed from yellow-orange to yellow-green, so we could expect that the birefringence changes. Moreover, the extinction position rotated clockwise and anticlockwise depending on polarity of the applied field (Figure 3). Such texture transformation is characteristic for a transition from the $\mathrm{SmC}_{\mathrm{A}} \mathrm{P}_{\mathrm{A}}$ phase to the $\mathrm{SmC}_{\mathrm{S}} \mathrm{P}_{\mathrm{F}}$ phase under the electric field. In the $\mathrm{SmC}_{\mathrm{S}} \mathrm{P}_{\mathrm{F}}$, all molecules are turned towards polarity of the applied field. The electro-optical behavior for the $\mathrm{SmC}_{\mathrm{A}} \mathrm{P}_{\mathrm{A}}-\mathrm{SmC}_{\mathrm{S}} \mathrm{P}_{\mathrm{F}}$ transition has been described in literature [2,11,12], schematically, we demonstrated such a type of molecular reorientation in Figure 4. We expect that the molecules rotate on the conus and do not change the chirality.

Table 1. Melting point, m.p., the phase transition temperature from the isotropic (Iso) phase to the mesophase, $T_{\mathrm{iso}}$, and temperature of crystallization, $T_{\mathrm{cr}}$, in ${ }^{\circ} \mathrm{C}$, and corresponding enthalpy changes, $\Delta H$ in $\mathrm{kJ} \mathrm{mol}^{-1}$, detected on the second temperature runs, are in brackets.

\begin{tabular}{cccccccc}
\hline Comp. & $\mathbf{X}$ & $\mathbf{R}$ & $\begin{array}{c}\text { m.p. } \\
\boldsymbol{\Delta} \boldsymbol{H}\end{array}$ & $\begin{array}{c}\boldsymbol{T}_{\mathbf{c r}} \\
\boldsymbol{\Delta} \boldsymbol{H}\end{array}$ & $\mathbf{M}$ & $\begin{array}{c}\boldsymbol{T}_{\text {iso }} \\
\boldsymbol{H}\end{array}$ & Iso \\
\hline Ia & $\mathrm{F}$ & $\mathrm{C}_{8} \mathrm{H}_{17}$ & $123(+50.0)$ & $104(-29.6)$ & $\mathrm{B}_{1}$ & $121(-50.0)$ & $\bullet$ \\
\hline Ib & $\mathrm{F}$ & $\mathrm{C}_{10} \mathrm{H}_{21}$ & $88(+18.7)$ & $75(-16.6)$ & $\mathrm{B}_{1}$ & $101(-18.8)$ & $\bullet$ \\
\hline Ic & $\mathrm{F}$ & $\mathrm{C}_{12} \mathrm{H}_{25}$ & $95(+31.9)$ & $83(-33.5)$ & $\mathrm{SmC}_{\mathrm{A}} \mathrm{P}_{\mathrm{A}}$ & $96(-19.1)$ & $\bullet$ \\
\hline Id & $\mathrm{F}$ & $\mathrm{C}_{14} \mathrm{H}_{29}$ & $95(+44.3)$ & $90(-44.3)$ & $\mathrm{SmC}_{\mathrm{A}} \mathrm{P}_{\mathrm{A}}$ & $100(-21.1)$ & $\bullet$ \\
\hline IIa & $\mathrm{Cl}$ & $\mathrm{C}_{8} \mathrm{H}_{17}$ & $118(+45.7)$ & $101(-44.0)$ & - & - & $\bullet$ \\
\hline
\end{tabular}


Table 1. Cont.

\begin{tabular}{cccccccc}
\hline Comp. & $\mathbf{X}$ & $\mathbf{R}$ & $\begin{array}{c}\mathbf{m} . \mathbf{p} . \\
\boldsymbol{H}\end{array}$ & $\begin{array}{c}\boldsymbol{T}_{\mathbf{c r}} \\
\boldsymbol{H} \boldsymbol{H}\end{array}$ & $\mathbf{M}$ & $\begin{array}{c}\boldsymbol{T}_{\text {iso }} \\
\boldsymbol{H} \boldsymbol{H}\end{array}$ & Iso \\
\hline IIb & $\mathrm{Cl}$ & $\mathrm{C}_{10} \mathrm{H}_{21}$ & $107(+40.0)$ & $100(-37.6)$ & - & - & $\bullet$ \\
\hline IIc & $\mathrm{Cl}$ & $\mathrm{C}_{12} \mathrm{H}_{25}$ & $106(+41.0)$ & $101(-37.4)$ & - & - & $\bullet$ \\
\hline IId & $\mathrm{Cl}$ & $\mathrm{C}_{14} \mathrm{H}_{29}$ & $107(+50.1)$ & $102(-42.8)$ & - & - & $\bullet$ \\
\hline IIIa & $\mathrm{CH}_{3}$ & $\mathrm{C}_{8} \mathrm{H}_{17}$ & $125(+45.3)$ & $103(-44.9)$ & - & - & $\bullet$ \\
\hline IIIb & $\mathrm{CH}_{3}$ & $\mathrm{C}_{10} \mathrm{H}_{21}$ & $108(+66.1)$ & $100(-32.6)$ & - & - & $\bullet$ \\
\hline IIIc & $\mathrm{CH}_{3}$ & $\mathrm{C}_{12} \mathrm{H}_{25}$ & $88(+35.9)$ & $99(-25.1)$ & - & - & $\bullet$ \\
\hline IIId & $\mathrm{CH}_{3}$ & $\mathrm{C}_{14} \mathrm{H}_{29}$ & $66(+11.4)$ & $65(-14.2)$ & $\mathrm{CrX}$ & $103(-25.9)$ & $\bullet$ \\
\hline IVa & $\mathrm{NO}_{2}$ & $\mathrm{C}_{8} \mathrm{H}_{17}$ & $127(+39.1)$ & $125(-37.4)$ & - & - & $\bullet$ \\
\hline IVb & $\mathrm{NO}_{2}$ & $\mathrm{C}_{10} \mathrm{H}_{21}$ & $129(+42.1)$ & $126(-41.9)$ & - & - & $\bullet$ \\
\hline IVc & $\mathrm{NO}_{2}$ & $\mathrm{C}_{12} \mathrm{H}_{25}$ & $132(+44.2)$ & $128(-42.6)$ & - & - & $\bullet$ \\
\hline IVd & $\mathrm{NO}_{2}$ & $\mathrm{C}_{14} \mathrm{H}_{29}$ & $129(+47.2)$ & $126(-48.6)$ & - & - & $\bullet$ \\
\hline
\end{tabular}

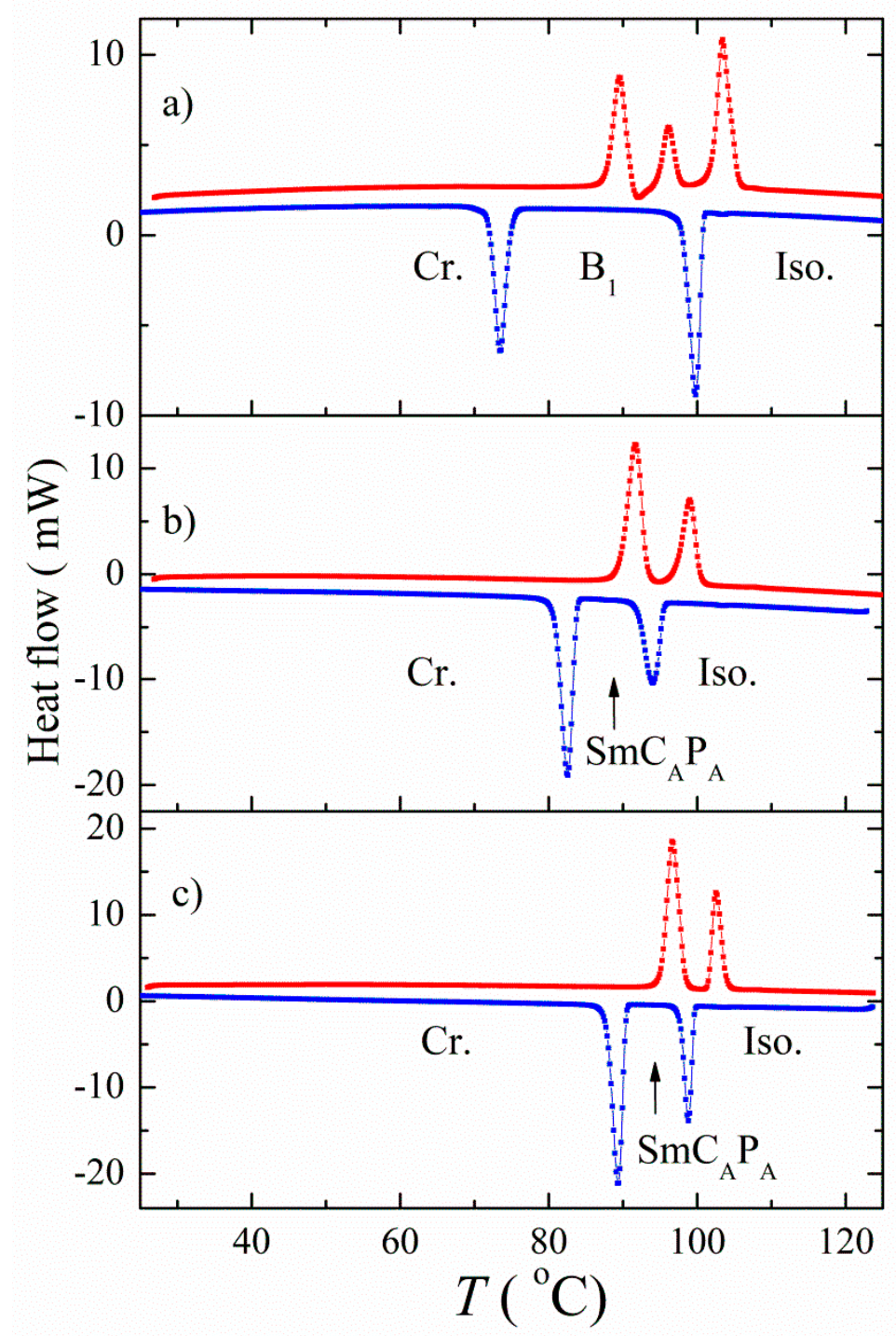

Figure 1. Thermographs taken during the second DSC runs for (a) Ib, (b) Ic, and (c) Id. Red colors are for the second heating and the blue color for the second cooling runs. 


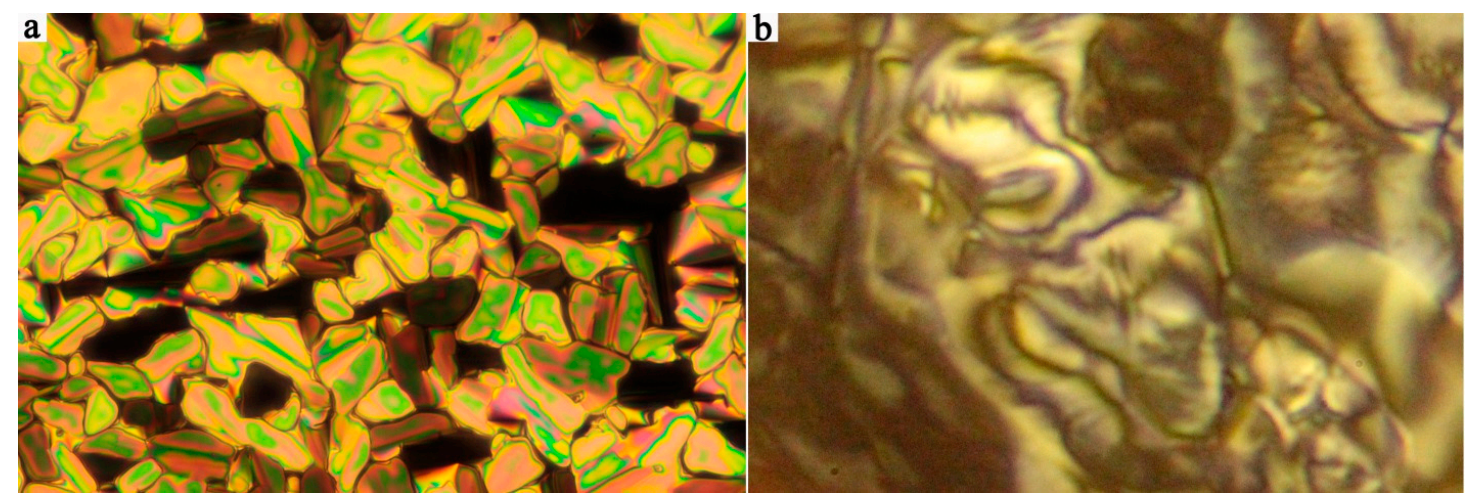

Figure 2. (a) Planar texture of $I a$ in the $B_{1}$ phase at $T=115^{\circ} \mathrm{C}$, the width of the photo is about $200 \mu \mathrm{m}$. (b) Sample with free upper surface with a schlieren texture for Ic in the $\mathrm{SmC}_{\mathrm{A}} \mathrm{P}_{\mathrm{A}}$ phase at $\mathrm{T}=92{ }^{\circ} \mathrm{C}$. Width of the photos corresponds to $150 \mu \mathrm{m}$.

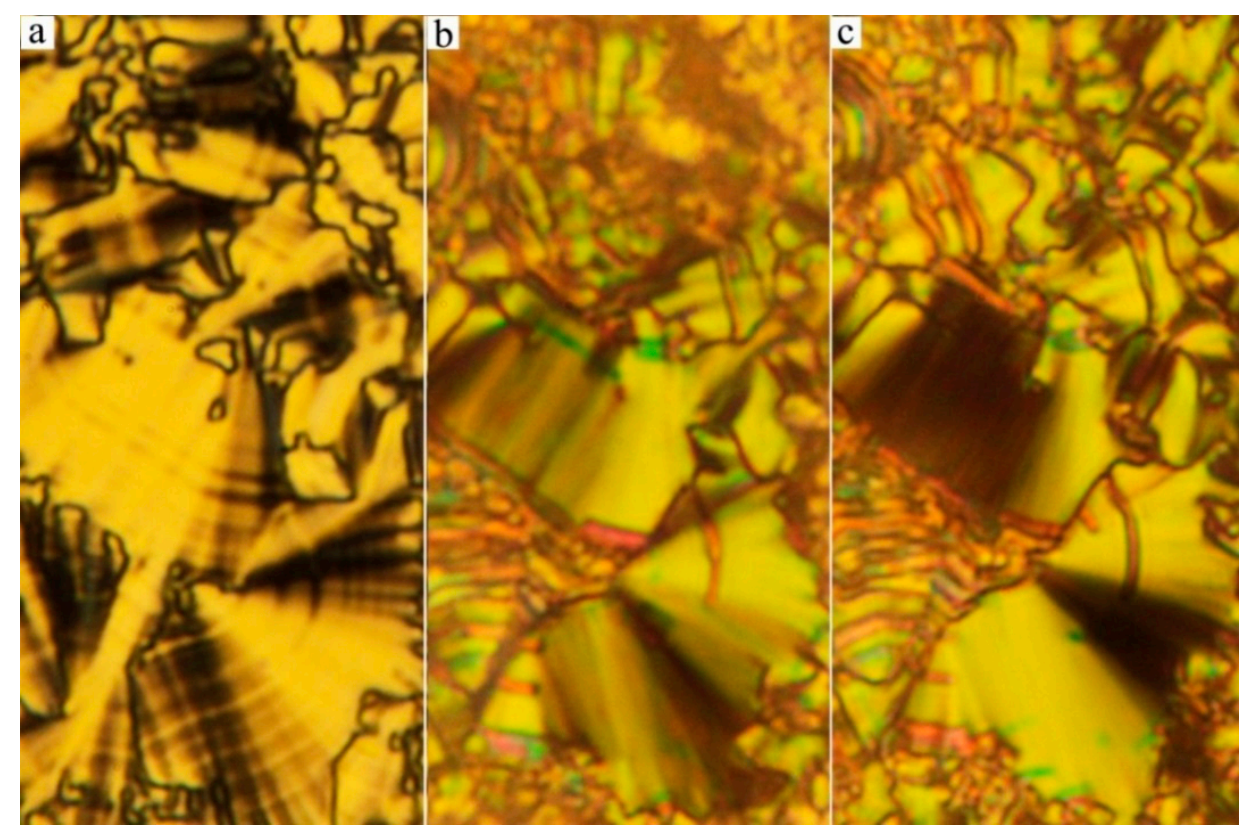

Figure 3. Textures in the $S m C_{A} P_{A}$ phase of Id at $\mathrm{T}=96{ }^{\circ} \mathrm{C}$ : (a) without the applied electric field, (b) and (c) under the field of about $\pm 10 \mathrm{~V} / \mu \mathrm{m}$, with opposite polarity, respectively. Polarizers are oriented along the edge of the photo, the width of each photo corresponds to about $100 \mu \mathrm{m}$.

We studied a polarization current in the $\mathrm{SmC}_{\mathrm{A}} \mathrm{P}_{\mathrm{A}}$ phase. We found that there were two peaks per half-cycle in the profile of the polarization current, which was induced in an a.c. field of the triangular shape. The switching current is demonstrated in Figure 5a,b for Ic and Id, respectively. Under a sufficiently large applied electric field, higher than $10 \mathrm{~V} / \mu \mathrm{m}$, we detected polarization, $P(\mathrm{~T})$, which did not change with temperature within the $\mathrm{SmC}_{\mathrm{A}} \mathrm{P}_{\mathrm{A}}$ phase on cooling and $P$ values reached about $500 \mathrm{nC} / \mathrm{cm}^{2}$ and $700 \mathrm{nC} / \mathrm{cm}^{2}$ for Ic and Id, respectively. 


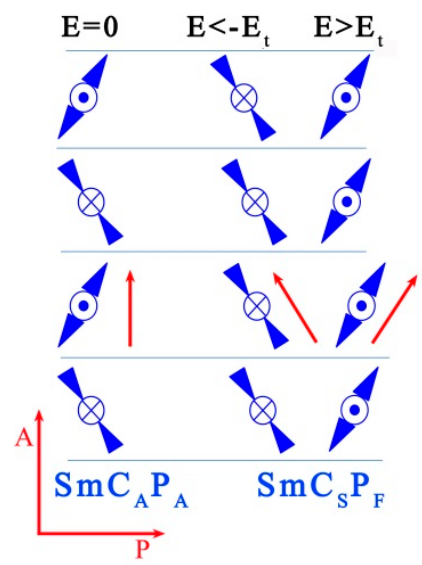

Figure 4. Schematic picture of molecular organization in neighbouring layers: the first column shows the original $\mathrm{SmC}_{\mathrm{A}} \mathrm{P}_{\mathrm{A}}$ phase, the second and the third column demonstrate the reorientation of molecules in the applied electric field $+\mathrm{E}$ and $-\mathrm{E}$ in the $\mathrm{SmC}_{\mathrm{S}} \mathrm{P}_{\mathrm{F}}$ phase. The perpendicular arrows in the bottom left corner show the orientation of polarizers, and the red arrows mark the orientation of the average molecular axis.
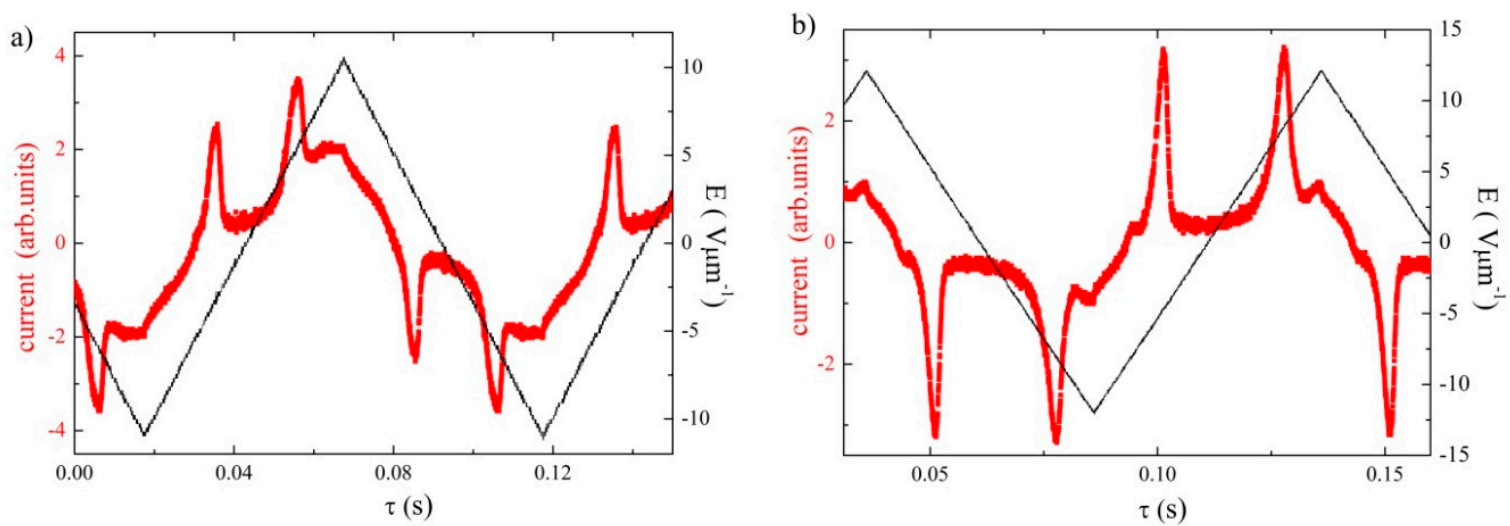

Figure 5. Switching current profile for (a) Ic at $\mathrm{T}=95^{\circ} \mathrm{C}$, and (b) for $\mathbf{I d}$ at $\mathrm{T}=96^{\circ} \mathrm{C}$.

\subsection{Dielectric Spectroscopy}

Dielectric properties in the $\mathrm{SmC}_{\mathrm{A}} \mathrm{P}_{\mathrm{A}}$ phase were studied in detail. The complex permittivity was acquired in the frequency range from $100 \mathrm{~Hz}$ to $10 \mathrm{MHz}$ on cooling from the isotropic phase. We detected a weak high-frequency mode, which was present only in the temperature range of the $\mathrm{SmC}_{\mathrm{A}} \mathrm{P}_{\mathrm{A}}$ phase. Three-dimensional (3D) graphs of the imaginary part of the permittivity, $\varepsilon^{\prime \prime}$, are shown in Figure 6 for compounds Ic and Id, on cooling from the isotropic phase, through the $\mathrm{SmC}_{\mathrm{A}} \mathrm{P}_{\mathrm{A}}$ phase down to the crystallization. The relaxation mode was present only in the temperature range of the $\mathrm{SmC}_{\mathrm{A}} \mathrm{P}_{\mathrm{A}}$ phase and it disappeared in the isotropic as well as in the crystalline phases. The mode can be attributed to the collective mode, which is often present in SmCP phases, which is probably related to the antiferroelectric character of the mesophase. We analyzed the dielectric behavior with respect to Cole-Cole formula (1) and obtained the relaxation frequency, $f_{r}$, and the dielectric strength, $\Delta \varepsilon$, in the $\mathrm{SmC}_{\mathrm{A}} \mathrm{P}_{\mathrm{A}}$ phase. Temperature dependency of the relaxation frequency, $f_{r}$, and the dielectric strength, $\Delta \varepsilon$, for Ic and Id are shown in Figure 7 . We found $f_{r}(\mathrm{~T})$ decreased continuously on cooling and we obtained an activation energy when fitted to the Arrhenius law. 

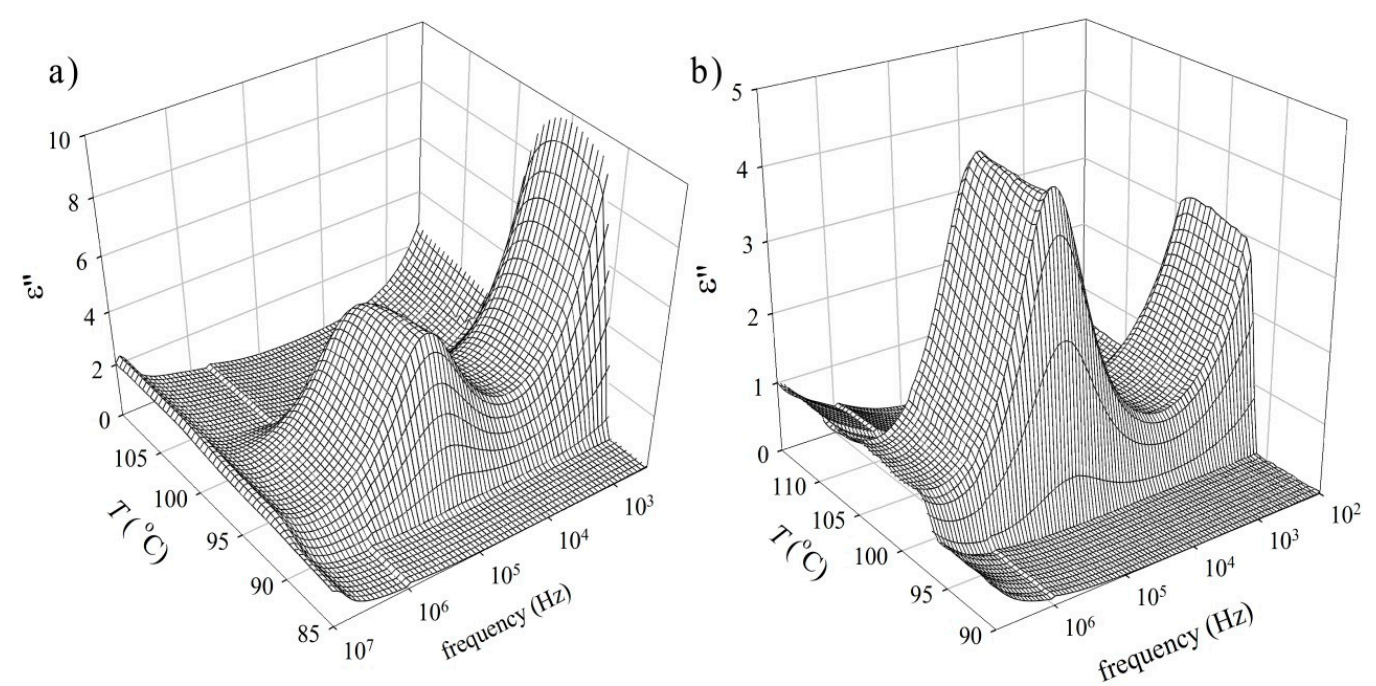

Figure 6. Dielectric spectroscopy results: the imaginary part of permittivity, $\varepsilon^{\prime \prime}$, versus frequency and temperature, $T$, for compounds (a) Ic, and (b) Id.

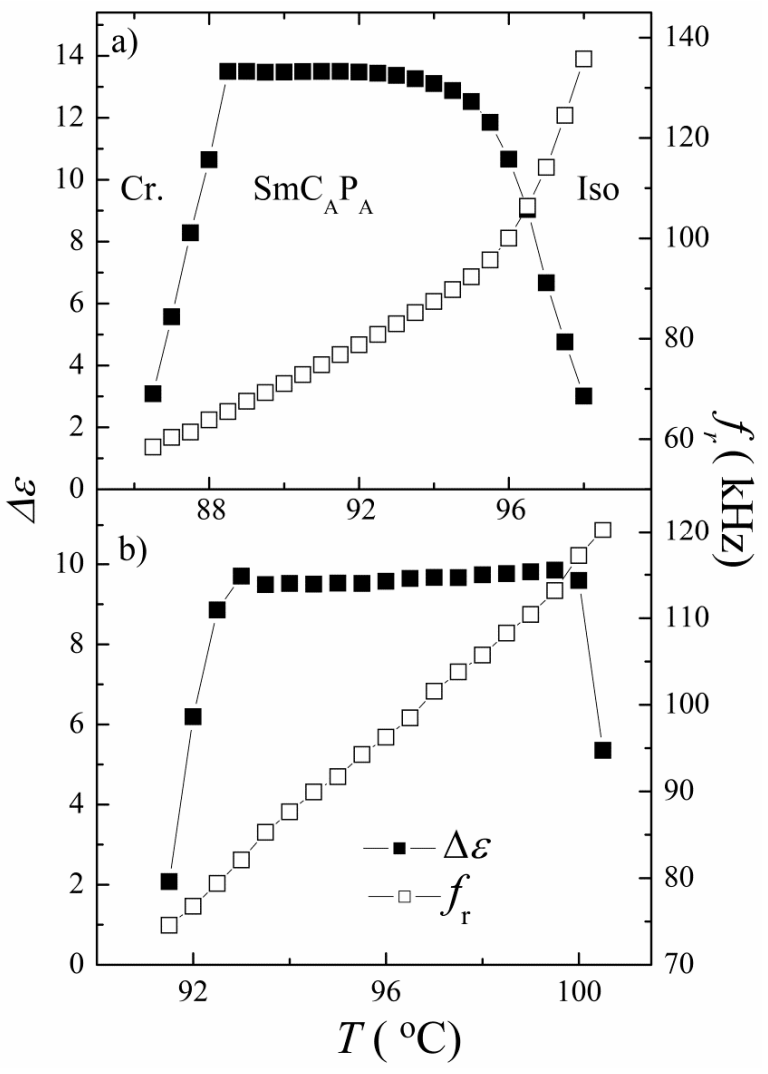

Figure 7. Temperature dependencies of the relaxation frequency, $f_{r}$, and the dielectric strength, $\Delta \varepsilon$, in the $\mathrm{SmC}_{\mathrm{A}} \mathrm{P}_{\mathrm{A}}$ phase for (a) Ic, and (b) Id.

\subsection{X-ray Measurements}

X-ray scattering measurements were performed for all mesogenic compounds from the series I. For compounds Ia and $\mathbf{I b}$ in the small-angle region, the diffractograms exhibited incommensurate reflections (Figure 8a) that can be indexed assuming centred rectangular unit cells. This type of diffraction pattern is characteristic for a $\mathrm{B}_{1}$-type of columnar mesophases. For $\mathbf{I a}$, the unit cell at $\mathrm{T}=114{ }^{\circ} \mathrm{C}$ was calculated and we established $\mathrm{a}=32.5 \AA$ and $\mathrm{b}=41.8 \AA$. For $\mathbf{I b}$ at $\mathrm{T}=100{ }^{\circ} \mathrm{C}$, $\mathrm{a}=43.8 \AA$ and $\mathrm{b}=44.2 \AA$ were found. The parameter $b$ of the unit cell is related to the molecular 
length and it reflects the extension of the terminal chains, when we compare Ia and $\mathbf{I b}$. For Ic and Id in the $\mathrm{SmC}_{\mathrm{A}} \mathrm{P}_{\mathrm{A}}$ phase, the XRD signal in the small-angle region revealed sharp commensurate peaks, which corresponded to the smectic layers. The layer spacing value, $d$, was calculated and for Ic we found $\mathrm{d}=40.3 \AA$ (at the temperature $\mathrm{T}=95^{\circ} \mathrm{C}$ ) and for Id $\mathrm{d}=42.2 \AA$ (at $\mathrm{T}=99^{\circ} \mathrm{C}$ ). For all studied mesogenic compounds, the diffuse high-angle maxima correspond to about $4.5 \AA$, which is the averaged intermolecular distance within layers. In Figure 8, we present an XRD intensity profile with respect to the scattering angle for two selected compounds. In Figure 8a, there is the intensity versus scattering angle for $\mathbf{I b}$ in the $\mathrm{B}_{1}$ phase with Miller indexes at the corresponding peaks. In Figure $8 \mathbf{b}$, the intensity versus scattering angle is demonstrated for Ic. In Table 2, crystallographic parameters are summarized and compared with the length of the molecule, $l$, obtained from ab initio calculations. The tilt angle of molecules with respect to the layer normal, $\gamma$, can be calculated and we calculated it for both types of mesophases, the columnar $\mathrm{B}_{1}$ phase as well as in the $\mathrm{SmC}_{\mathrm{A}} \mathrm{P}_{\mathrm{A}}$ phase (Table 2). In the $\mathrm{SmC}_{\mathrm{A}} \mathrm{P}_{\mathrm{A}}$ phase, the value of the tilt angle was approaching 45 degrees, which was in agreement with observations of textures under the applied electric field, as we observed rotation of the extinction position for an angle of $42-45$ degrees.
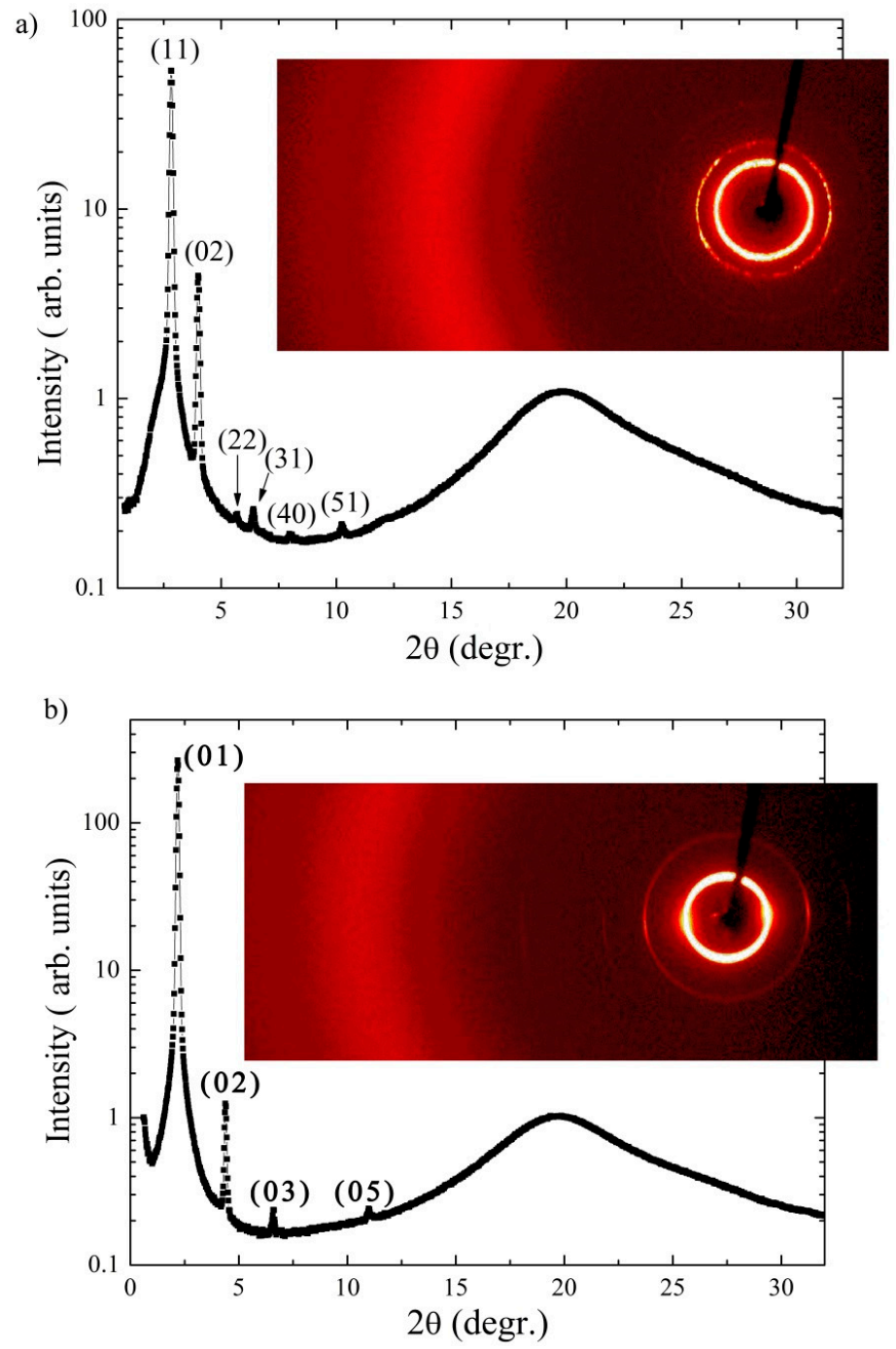

Figure 8. X-ray intensity profile with respect to the scattering angle (a) for $\mathbf{I b}$ in the $\mathrm{B}_{1}$ phase at $\mathrm{T}=100{ }^{\circ} \mathrm{C}$, and (b) for Ic at $\mathrm{T}=95^{\circ} \mathrm{C}$. Miller indexes are placed above the corresponding peaks. 
Table 2. Parameters of the crystallographic unit cell, $a$ and $b$, at selected temperatures, $T$. For Ic and Id the layer spacing, $d$, and the calculated length of the molecule, $l$, and calculated value of the tilt angle, $\gamma$, are presented.

\begin{tabular}{ccccccc}
\hline & $\mathbf{T} /{ }^{\circ} \mathbf{C}$ & $a / \AA$ & $\mathbf{b} / \AA$ & $d / \AA$ & $l / \AA$ & $\gamma / \mathbf{d e g r}$. \\
\hline Ia & 114 & 32.5 & 41.8 & & 45.8 & 24 \\
\hline Ib & 100 & 43.8 & 44.2 & & 50.1 & 28 \\
\hline Ic & 95 & & & 40.3 & 54.5 & 42.3 \\
\hline Id & 99 & & & 42.2 & 58.9 & 44.2 \\
\hline
\end{tabular}

\section{Discussion and Conclusions}

In this study, we have focused on the mesomorphic behavior of bent-core liquid crystals bearing the lateral substituent in the apex position of the core. Previously, we documented for derivatives of 3-hydroxybenzoic acid with lateral substituents in position six that only materials bearing the smallest substituent (fluorine) exhibited mesomorphic behavior while other substituents caused crystallinity of the substances [34]. In this particular case, the mesomorphic behavior was highly dependent on the number and orientation of the ester linkages. Tuning the orientation of the ester groups (Figure 9a), it was possible to induce the formation of an enantiotropic columnar $\mathrm{B}_{1}$ phase and lamellar $\mathrm{SmC}_{\mathrm{A}} \mathrm{P}_{\mathrm{A}}$ phases. In the case of methyl and chlorine as the substituents in the apex position, only monotropic $B_{1}$ and an enantiotropic $S_{m} C_{A} P_{A}$ phase for the materials with longest terminal alkyl chain $\left(\mathrm{C}_{14} \mathrm{H}_{29}\right)$ were observed. Subsequently, this plausible orientation of ester linkages was adopted also for the materials studied here. Despite the optimum number and orientation of the ester linking units, only the fluoro-substituted materials I exhibited mesomorphic properties. It is reasonable to assume that in the case of materials of series II-IV, a negative steric effect of larger substituents hinders the formation of a mesophase. Similar behavior has already been discussed for 5-substituted resorcinol-based materials (1,3-phenylene bis[4-(4-alkyloxyphenoxycarbonyl)benzoates, (Figure $9 \mathrm{~b}$ ) for which all studied fluoro-substituted homologues exhibited a $\mathrm{SmCP}_{\mathrm{A}}$ phase, while other lateral substituents were not tolerated [29]. It should be noted that the electronic effect of the substituents is less likely to affect the physical properties of the materials. The lateral substituents are located in meta-position to the functional groups connecting the elongating side arms and, thus, cannot significantly affect their electronic state and, consequently, their conformation.

In this contribution we have synthesized central cores laterally substituted in the apex position and applied them in the synthesis of four series of novel bent-core liquid crystals. From the DSC studies and texture observation under an optical polarizing microscope, we have found that only the materials of the series I show mesomorphic behavior, while materials of series II-IV bearing bulky substituents are crystalline only. Using electro-optical investigations, dielectric spectroscopy, and $\mathrm{X}$-ray measurements, we have determined the character of the mesophases. We show that the fluoro-substituted homologues with the shorter terminal alkyl chains $\left(\mathrm{C}_{8} \mathrm{H}_{17}\right.$ and $\left.\mathrm{C}_{10} \mathrm{H}_{21}\right)$ exhibit the columnar $\mathrm{B}_{1}$ phase while materials with longer terminal alkyl chains $\left(\mathrm{C}_{12} \mathrm{H}_{25}\right.$ and $\left.\mathrm{C}_{14} \mathrm{H}_{29}\right)$ show the $\mathrm{SmC}_{\mathrm{A}} \mathrm{P}_{\mathrm{A}}$ phase.

It can be concluded that the mesomorphic behavior of the materials substituted in the apex position strongly depends on the size of the lateral substituent with fluorine being most probably the only tolerable one. The mesomorphic properties of the fluoro-substituted materials can be tuned by the length of the terminal alkyl chains. However, the effect of the type, number, and orientation of the linking units in the side chains is yet to be studied in detail. 
<smiles>[R2]Oc1ccc(OC(=O)c2ccc(OC(=O)c3ccc(OC(=O)c4ccc(OC(=O)c5ccc([R20])cc5)cc4)cc3)c([X])c2)cc1</smiles>

a R $=\mathrm{C}_{8} \mathrm{H}_{17}, \mathbf{b} \mathrm{R}=\mathrm{C}_{10} \mathrm{H}_{21}, \mathbf{c} \mathrm{R}=\mathrm{C}_{12} \mathrm{H}_{25}, \mathbf{d} \mathrm{R}=\mathrm{C}_{14} \mathrm{H}_{29}$<smiles>[R]Oc1ccc(C(=O)Oc2ccc(C(=O)Oc3cc(F)cc(OC(=O)c4ccc(OC(=O)c5ccc([R])cc5)cc4)c3)cc2)cc1</smiles>

Figure 9. The structure of (a) materials based on 6-substituted 3-hydroxybenzoic acid studied in reference [34], and (b) materials derived from 5-substituted resorcinol studied in reference [29].

Author Contributions: Conceptualization, J.S. and M.K.; Methodology, J.S, V.N. and V.K.; Formal analysis, V.N., D.P., H.S. and P.Š.; Investigation, H.S., P.Š., D.P. and V.N.; Data curation, J.S., V.N. and D.P.; Writing-original draft preparation, J.S., M.K. and V.N.; Writing-review and editing, J.S., M.K. and V.N.; Funding acquisition, J.S. and V.N. All authors have read and agreed to the published version of the manuscript.

Funding: This work was supported by the Czech Ministry of Education, Youth and Sports [project number LTC19051-MEYS], Operational Programme Research, Development and Education financed by European Structural and Investment Funds [project No. SOLID21-CZ.02.1.01/0.0/0.0/16_019/0000760], and the Czech Science Foundation (project No. 18-14497S).

Conflicts of Interest: No potential conflict of interest was reported by the authors.

\section{References}

1. Vorländer, D. Die Richtung der Kohlenstoff-Valenzen in Benzol-Abkömmlingen. Ber. Dtsch. Chem. Ges. 1929, 62, 2831-2835. [CrossRef]

2. $\quad$ Link, D.R.; Natale, G.; Shao, R.; Maclennan, J.E.; Clark, N.A.; Körblova, E.; Walba, D.M. Spontaneous Formation of Macroscopic Chiral Domains in a Fluid Smectic Phase of Achiral Molecules. Science 1997, 278, 1924-1927. [CrossRef] [PubMed]

3. Niori, T.; Sekine, T.; Watanabe, J.; Furukawa, T.; Takezoe, H. Distinct ferroelectric smectic liquid crystals consisting of banana shaped achiral molecules. J. Mater. Chem. 1996, 6, 1231-1233. [CrossRef]

4. Hideo, T.; Yoichi, T. Bent-Core Liquid Crystals: Their Mysterious and Attractive World. Jpn. J. Appl. Phys. 2006, 45, 597-625. [CrossRef]

5. Jákli, A. Liquid crystals of the twenty-first century-nematic phase of bent-core molecules. Liq. Cryst. Rev. 2013, 1, 65-82. [CrossRef]

6. Madsen, L.A.; Dingemans, T.J.; Nakata, M.; Samulski, E.T. Thermotropic Biaxial Nematic Liquid Crystals. Phys. Rev. Lett. 2004, 92, 145505. [CrossRef]

7. Tschierske, C.; Photinos, D.J. Biaxial nematic phases. J. Mater. Chem. 2010, 20, 4263-4294. [CrossRef]

8. Pelzl, G.; Eremin, A.; Diele, S.; Kresse, H.; Weissflog, W. Spontaneous chiral ordering in the nematic phase of an achiral banana-shaped compound. J. Mater. Chem. 2002, 12, 2591-2593. [CrossRef]

9. Vita, F.; Adamo, F.C.; Francescangeli, O. Polar order in bent-core nematics: An overview. J. Mol. Liq. 2018. [CrossRef] 
10. Connor, P.L.M.; Mandle, R.J. Chemically induced splay nematic phase with micron scale periodicity. Soft Matter 2020, 16, 324-329. [CrossRef]

11. Jing, H.; Xu, M.; Xiang, Y.; Wang, E.; Liu, D.; Poryvai, A.; Kohout, M.; Éber, N.; Buka, Á. Light Tunable Gratings Based on Flexoelectric Effect in Photoresponsive Bent-Core Nematics. Adv. Opt. Mater. 2019, 7, 1801790. [CrossRef]

12. Reddy, R.A.; Tschierske, C. Bent-core liquid crystals: Polar order, superstructural chirality and spontaneous desymmetrisation in soft matter systems. J. Mater. Chem. 2006, 16, 907-961. [CrossRef]

13. Tschierske, C. Development of Structural Complexity by Liquid-Crystal Self-assembly. Angew. Chem. Int. Ed. 2013, 52, 8828-8878. [CrossRef] [PubMed]

14. Etxebarria, J.; Blanca Ros, M. Bent-core liquid crystals in the route to functional materials. J. Mater. Chem. 2008, 18, 2919-2926. [CrossRef]

15. Pelzl, G.; Diele, S.; Weissflog, W. Banana-Shaped Compounds-A New Field of Liquid Crystals. Adv. Mater. 1999, 11, 707-724. [CrossRef]

16. Shen, D.; Pegenau, A.; Diele, S.; Wirth, I.; Tschierske, C. Molecular Design of Nonchiral Bent-Core Liquid Crystals with Antiferroelectric Properties. J. Am. Chem. Soc. 2000, 122, 1593-1601. [CrossRef]

17. Weissflog, W.; Naumann, G.; Kosata, B.; Schroder, M.W.; Eremin, A.; Diele, S.; Vakhovskaya, Z.; Kresse, H.; Friedemann, R.; Krishnan, S.A.R.; et al. Ten isomeric five-ring bent-core mesogens: The influence of the direction of the carboxyl connecting groups on the mesophase behaviour. J. Mater. Chem. 2005, 15, 4328-4337. [CrossRef]

18. Kohout, M.; Svoboda, J.; Novotná, V.; Glogarová, M.; Pociecha, D. Non-symmetrical bent-shaped liquid crystals with five ester groups. Liq. Cryst. 2010, 37, 987-996. [CrossRef]

19. Kohout, M.; Svoboda, J.; Novotná, V.; Pociecha, D. Non-symmetrical bent-shaped liquid crystals based on a laterally substituted naphthalene central core with four ester groups. Liq. Cryst. 2011, 38, 1099-1110. [CrossRef]

20. Kohout, M.; Svoboda, J.; Novotná, V.; Pociecha, D.; Glogarová, M.; Gorecka, E. A nematic-polar columnar phase sequence in new bent-shaped liquid crystals based on a 7-hydroxynaphthalene-2-carboxylic acid core. J. Mater. Chem. 2009, 19, 3153-3160. [CrossRef]

21. Amaranatha Reddy, R.; Baumeister, U.; Keith, C.; Hahn, H.; Lang, H.; Tschierske, C. Influence of the core structure on the development of polar order and superstructural chirality in liquid crystalline phases formed by silylated bent-core molecules: Lateral substituents. Soft Matter 2007, 3, 558-570. [CrossRef]

22. Kovalenko, L.; Weissflog, W.; Grande, S.; Diele, S.; Pelzl, G.; Wirth, I. Dimorphism SmA-B2 in bent-core mesogens with perfluorinated terminal chains. Liq. Cryst. 2000, 27, 683-687. [CrossRef]

23. Alaasar, M.; Prehm, M.; Tschierske, C. Influence of halogen substituent on the mesomorphic properties of five-ring banana-shaped molecules with azobenzene wings. Liq. Cryst. 2013, 40, 656-668. [CrossRef]

24. Bedel, J.P.; Rouillon, J.C.; Marcerou, J.P.; Laguerre, M.; Nguyen, H.T.; Achard, M.F. Influence of fluoro substituents on the mesophase behaviour of banana-shaped molecules. J. Mater. Chem. 2002, 12, 2214-2220. [CrossRef]

25. Dantlgraber, G.; Keith, C.; Baumeister, U.; Tschierske, C. Polar and chiral mesophases formed by m-terphenyl derived bent-core molecules with lateral F-substituents. J. Mater. Chem. 2007, 17, 3419-3426. [CrossRef]

26. Nadasi, H.; Weissflog, W.; Eremin, A.; Pelzl, G.; Diele, S.; Das, B.; Grande, S. Ferroelectric and antiferroelectric "banana phases" of new fluorinated five-ring bent-core mesogens. J. Mater. Chem. 2002, 12, 1316-1324. [CrossRef]

27. Svoboda, J.; Novotna, V.; Kozmik, V.; Glogarova, M.; Weissflog, W.; Diele, S.; Pelzl, G. A novel type of banana liquid crystals based on 1-substituted naphthalene-2,7-diol cores. J. Mater. Chem. 2003, 13, $2104-2110$. [CrossRef]

28. Weissflog, W.; Nadasi, H.; Dunemann, U.; Pelzl, G.; Diele, S.; Eremin, A.; Kresse, H. Influence of lateral substituents on the mesophase behaviour of banana-shaped mesogens. J. Mater. Chem. 2001, 11, 2748-2758. [CrossRef]

29. Weissflog, W.; Shreenivasa Murthy, H.N.; Diele, S.; Pelzl, G. Relationships between molecular structure and physical properties in bent-core mesogens. Philos. Trans. R. Soc. A 2006, 364, 2657-2679. [CrossRef]

30. Umadevi, S.; Radhika, S.; Sadashiva, B.K. The SmCPA phase in five-ring bent-core compounds derived from 5-methoxyisophthalic acid. Liq. Cryst. 2006, 33, 139-147. [CrossRef]

31. Kozmík, V.; Polášek, P.; Seidler, A.; Kohout, M.; Svoboda, J.; Novotná, V.; Glogarová, M.; Pociecha, D. The effect of a thiophene ring in the outer position on mesomorphic properties of the bent-shaped liquid crystals. J. Mater. Chem. 2010, 20, 7430-7435. [CrossRef] 
32. Kohout, M.; Kozmík, V.; Slabochová, M.; Tůma, J.; Svoboda, J.; Novotná, V.; Pociecha, D. Bent-shaped liquid crystals based on 4-substituted 3-hydroxybenzoic acid central core. Liq. Cryst. 2015, 42, 87-103. [CrossRef]

33. Tůma, J.; Kohout, M.; Svoboda, J.; Novotná, V.; Pociecha, D. Bent-shaped liquid crystals based on 4-substituted 3-hydroxybenzoic acid central core-Part II. Liq. Cryst. 2016, 43, 547-563. [CrossRef]

34. Tůma, J.; Kohout, M.; Svoboda, J.; Novotná, V.; Pociecha, D. Bent-core liquid crystals based on 6-substituted 3-hydroxybenzoic acid: The role of substitution and linkage group orientation on mesomorphic properties. Liq. Cryst. 2016, 43, 1889-1900. [CrossRef]

35. Pallová, L.; Kozmík, V.; Kohout, M.; Svoboda, J.; Novotná, V.; Pociecha, D. Bent-core liquid crystals with a 2-substituted 3-hydroxybenzoic acid central core. Liq. Cryst. 2017, 44, 1306-1315. [CrossRef]

36. Skopalová, H.; Kozmík, V.; Šmahel, M.; Svoboda, J.; Pacherová, O.; Kohout, M.; Novotná, V. Mesomorphic properties of non-symmetric bent-core liquid crystals with a lateral substituent in the apex position. Liq. Cryst. 2020, submitted.

37. Chamberland, S.; Grüschow, S.; Sherman, D.H.; Williams, R.M. Synthesis of Potential Early-Stage Intermediates in the Biosynthesis of FR900482 and Mitomycin C. Org. Lett. 2009, 11, 791-794. [CrossRef]

38. Richardson, S.K.; Jeganathan, A.; Mani, R.S.; Haley, B.E.; Watt, D.S.; Trusal, L.R. Synthesis and biological activity of C-4 and C-15 Aryl azide derivatives of anguidine. Tetrahedron 1987, 43, 2925-2934. [CrossRef]

39. Kohout, M.; Tuma, J.; Svoboda, J.; Novotna, V.; Gorecka, E.; Pociecha, D. 3-Hydroxycinnamic acid-a new central core for the design of bent-shaped liquid crystals. J. Mater. Chem. C 2013, 1, 4962-4969. [CrossRef]

40. Monte, M.J.S.; Almeida, A.R.R.P.; Matos, M.A.R. Thermodynamic Study on the Sublimation of Five Aminomethoxybenzoic Acids. J. Chem. Eng. Data 2010, 55, 419-423. [CrossRef]

41. Hartmann, R.W.; Heindl, A.; Schoenenberger, H. Ring-substituted 1,2-dialkylated 1,2-bis(hydroxyphenyl) ethanes. 2. Synthesis and estrogen receptor binding affinity of 4,4'-, 5, 5'-, and 6,6'-disubstituted metahexestrols. J. Med. Chem. 1984, 27, 577-585. [CrossRef]

(C) 2020 by the authors. Licensee MDPI, Basel, Switzerland. This article is an open access article distributed under the terms and conditions of the Creative Commons Attribution (CC BY) license (http://creativecommons.org/licenses/by/4.0/). 